\title{
Morphological Origins of Temperature and Rate Dependent Mechanical Properties of Model Soft Thermoplastic Elastomers
}

\author{
Simone Sbrescia ${ }^{1}$, Jianzhu Ju², Tom Engels ${ }^{1}$, Evelyne Van Ruymbeke ${ }^{3}$, Michelle Seitz ${ }^{1}$ \\ ${ }^{1}$ DSM Materials Science Center, Urmonderbaan, 6167 RD Geleen The Netherlands \\ ${ }^{2}$ Laboratoire Sciences et Ingénierie de la Matière Molle, ESPCI Paris, PSL University, CNRS, Sorbonne \\ Université, 75005 Paris, France \\ ${ }^{3}$ Bio and Soft Matter Division (BSMA), Institute of Condensed Matter and Nanosciences (IMCN), \\ Université Catholique de Louvain, B-1348 Louvain-la-Neuve, Belgium; \\ Correspondence to: Michelle Seitz (E-mail: michelle.seitz@dsm.com)
}

\begin{abstract}
Thermoplastic elastomers (TPEs) combine high elasticity with melt processability due to their structural features being based on physical associations rather than chemical crosslinking. Their mechanical properties are governed by the interplay of the different dynamics present in the system (i.e. hard block associations and soft block mobility) combined with their morphology. Irrespective of their exact chemical structure or type of association (crystals, hydrogen bonds, or glassy domains), many soft TPEs show a reduction in toughness at elevated temperatures. In this study, we investigate the high-temperature mechanical properties of a model series of industrially relevant TPEs via systematically varying composition and molecular weight. The results show an increase in temperature resistance and in large-strain stress response as chain length increases. We underline the key parameters that influence the mechanical behavior and explain the observed effect of molecular weight on both the temperature- and rate-dependent large-strain response. A physical network-based model is presented that can explain the experimental findings assuming an improved network connectivity and extended lifetime of the entangled segments with increasing molecular weight.
\end{abstract}

KEYWORDS: thermoplastic elastomer; morphology; thermomechanical property; network connectivity; strain recovery.

\section{INTRODUCTION}

Balancing high performance and recyclability with polymeric materials is a challenge. Rubbers with permanent chemical crosslinks are widely used because of their high flexibility, creep and chemical resistance as well as broad temperature range of use. However, they cannot be reshaped and reused. Replacing chemical crosslinks with non-permanent, physical associations enables both melt processing and recycling. Thermoplastic elastomers (TPEs) offer an alternative to rubbers as they are crosslinked via physical associations and trapped entanglements that lead to high elasticity coupled with melt processability. ${ }^{1}$ They are widely used in applications such as automotive ducts, shoes, and medical devices. However, TPEs are not yet able to replace cross-linked rubbers in all applications, as they generally have lower creep resistance and don't reach the same low-end values for the modulus. ${ }^{1}$ Some common examples of TPEs are segmented copolymers with associating blocks of polyesters, ${ }^{1-6}$ polyamides, ${ }^{1,7,8}$ and polyurethanes. ${ }^{9-11}$ They all 
share the feature of having a low glass transition $\left(\mathrm{T}_{\mathrm{g}}\right)$ of the soft blocks (SBs) combined with physically associating hard blocks (HBs). Their mechanical properties are governed by the interplay of the different dynamics present in the system (e.g. hard block associations and soft block mobility) as well as by their morphology, i.e. the physical arrangement of the polymer chains. ${ }^{1-7,9,10,12-18}$

For TPEs where the elasticity stems from crystallizing $\mathrm{HBs}$, the initial morphology develops upon cooling from the melt during which crystallization and phase separation occur. Typically, the assumption of only two dominant phases (soft amorphous and hard crystalline) is taken to simplify the interpretation of the mechanical results. ${ }^{4}$ Depending on the specific TPE, the fraction of associated HB can range from 20-80\%. ${ }^{19}$ Additionally the low Tg SB-rich phase may also be partially crystallized at lower temperatures. For many TPEs the morphology and, in consequence, mechanical properties evolve with deformation. Upon deformation, the initial crystal network breaks up into smaller domains that act as physical crosslinks. The microstructure evolution upon deformation at room temperature has been extensively studied for TPEs based on urethanes and esters (TPUs and TPE-Es respectively). . $7,8,18,20-24$ Complicating our further understanding is that their dynamics are strongly influenced by temperature, deformation and, for some chemistries, by strain induced crystallization of the initially mobile soft blocks. ${ }^{4,8,13,24}$

Previous studies mainly focused on the linear properties of TPE-Es with HB contents > 40 wt\%. ${ }^{4,5,13,14}$ The effects of the composition (SB/HB ratio) on the initial morphology and of the length of the individual blocks have been explored, showing increasing elastic modulus and melting temperature with increasing $\mathrm{HB}$ content. Systems with lower modulus and less temperature dependent rubber plateaus have been enabled by synthetic advances allowing mono-disperse hard blocks. ${ }^{10,18,25}$ However, most commercial TPU or TPE-E systems are based on polydisperse hard blocks.

Compared to rubber with permanent crosslinks, a major drawback of many TPEs based on multiblock copolymers with $<50 w t \% \mathrm{HB}$ is a large reduction in toughness at higher temperatures, independent of their exact chemical structure, type of association, or block polydispersity. ${ }^{18,26,27}$ This is a significant limitation in reaching low modulus, melt processible rubber alternatives with sufficiently broad temperature use range.

A few studies on TPE-Es and TPUs report mechanical data in the non-linear, large-strain regime. ${ }^{13,25-28}$ Currently lacking in the literature is a systematic study on the effects of the molecular weight $(\mathrm{Mw})$ on the mechanical properties as a function of temperature and strain-rate. To our knowledge, there are no studies combining both composition and temperature effects of TPE-Es. While there are a few observations, ${ }^{25,27}$ there is no systematic description of the influence of the $\mathrm{Mw}$ on the mechanical properties.

To gain insight into the key parameters that influence the mechanical behavior at different stages of deformation, we use combination of mechanical testing and morphology characterization to study the influence of composition (SB/HB ratio), temperature, and $\mathrm{Mw}$ for a series of model polyether-ester block copolymers. We emphasize here that despite the relatively small range in $\mathrm{Mw}$ among the studied samples, the effects on the high-strain 
mechanics are surprisingly large. Building upon the work of Aime et al. ${ }^{27}$ that explored the temperature dependent ultimate failure of softTPEs, we focus on what controls the level of stress at different temperatures and strainrates. The aim is to present a qualitative physical model that is able to explain the observations collected experimentally, taking into account the presence of both crystalline $\mathrm{HBs}$ and entanglements as stress bearing units as well as their time-temperature dependent kinetics.

This paper begins with a description of the model TPE-E systems' chemistry and composition. We then present the morphology of the system and how it evolves upon deformation, describing the linear and nonlinear mechanical properties focusing on the effects of composition, temperature, $\mathrm{Mw}$ and morphology evolution. Next, we present a first order physical picture that allows us to interpret the experimental results in light of local morphology and network connectivity and that is consistent with previous observations of similar materials. This picture is finally supported and enriched by a simple model that focuses on the $\mathrm{Mw}$ and rate effects and illustrates how dangling end disentanglement time influences the stress level. We end by drawing generic conclusions on the deformation mechanism of these model TPE-E that can be applied to a broad range of soft, segmented copolymer systems.

\section{EXPERIMENTAL}

\section{Materials}

We use as a model system a poly(ether-ester) block-copolymer based on polybutylene terephthalate (PBT) as $\mathrm{HB}$, and poly(tetramethylene oxide) (PTMO) as SB obtained via transesterification followed by polycondensation of dimethyl terephthalate (DMT), 1,4-butanediol (BDO) and PTMO diol. Details on the synthesis procedure have been previously described. ${ }^{4}$ After polymerization, the material is extruded and pelletized into granules.

The three different compositions studied here are reported in Table 1 . The soft blocks used had either a number average molecular weight, $\mathrm{M}_{\mathrm{n}, \mathrm{SB}}$, of $2 \mathrm{~kg} / \mathrm{mol}$ or $3 \mathrm{~kg} / \mathrm{mol}$ and they have a polydispersity index (PDI) of $\sim 2$. Materials are denoted by the wt\% and length of PTMO used followed by the total $M_{n}$ of the blockcopolymer, e.g. 60_PTMO2k_25 is a polymer with total $M_{n}$ of $25 \mathrm{~kg} / \mathrm{mol}$ made with $60 \mathrm{wt} \%$ of PTMO with $M_{n, S B}$ of $2 \mathrm{~kg} / \mathrm{mol}$. Different $M w$ are obtained via solid state post condensation (SSPC) for different times $(0,4,6,9,12$ hours) under dry nitrogen atmosphere at $180{ }^{\circ} \mathrm{C}$. For simplicity we refer to the amount of soft block in the system as the wt\% of PTMO (SB $\left.B_{w t}\right)$ used in the polymerization and the amount of hardblock as the wt fraction of PBT in the system $\left(H B_{w t}\right)$. It should be noted that the PTMO diols can be connected by a single DMT or by short DMT-BDO repeats that may not crystallize and thus act as chain extenders for the PTMO segments. It also follows that not all the DMT ends up in the $\mathrm{HBs}$ and $H B_{w t} \neq 1$ $\mathrm{SB}_{\mathrm{wt}} / 100$. We will simply refer to the segments comprising SBs and non-crystallized HBs as soft segments. By assuming random copolymerization of the components and calculating the molar fraction of PBT after polymerization, $X_{P B T}$, we can determine the average length of a $\mathrm{HB},\left\langle L_{n}\right\rangle$, expressed in terms of PBT repeat units, as follow: ${ }^{15}$

$<L_{n}>=\frac{1}{1-x_{P B T}}$, 
Equation 1 can be easily obtained from the definition of degree of polymerization, noting that for this system $X_{P B T}$ can be approximated to the propagation probability. This allows us to estimate the average number of HB per chain,

\begin{tabular}{|c|c|c|c|c|c|c|c|c|c|}
\hline Sample & $\begin{array}{l}\mathrm{SB}_{\mathrm{wt}} \\
{[\%]}\end{array}$ & $\begin{array}{c}\mathrm{M}_{\mathrm{n}, \mathrm{SB}} \\
{[\mathrm{kg} / \mathrm{mol}]^{\mathrm{a}}}\end{array}$ & $\begin{array}{c}\mathrm{T}_{\mathrm{m}} \text { peak } \\
{\left[{ }^{\circ} \mathrm{C}\right]^{\mathrm{b}}}\end{array}$ & $\begin{array}{c}X\left(X_{P B T}\right) \\
{[\%]^{c}}\end{array}$ & $\begin{array}{c}<L_{n}> \\
{[\#]^{\mathrm{d}}}\end{array}$ & $\begin{array}{l}<N> \\
{[\#]^{\mathrm{e}}}\end{array}$ & $\begin{array}{c}<N_{\text {crys }}> \\
{[\#]^{\dagger}}\end{array}$ & $\begin{array}{c}M_{n} \\
{[\mathrm{~kg} / \mathrm{mol}]^{\mathrm{g}}}\end{array}$ & $\begin{array}{c}M_{W} \\
{[\mathrm{~kg} / \mathrm{mol}]^{\mathrm{h}}}\end{array}$ \\
\hline \multirow{6}{*}{ 60_PTMO2k } & \multirow{6}{*}{60} & \multirow{6}{*}{2} & \multirow{6}{*}{200} & \multirow{6}{*}{$17(47)$} & \multirow{6}{*}{6.5} & 6.2 & 2.9 & 24.6 & 49.0 \\
\hline & & & & & & 7.5 & 3.5 & 29.4 & 59.1 \\
\hline & & & & & & 8.1 & 3.8 & 31.9 & 62.1 \\
\hline & & & & & & 8.7 & 4.1 & 34.2 & 67.0 \\
\hline & & & & & & 11.1 & 5.2 & 43.9 & 88.0 \\
\hline & & & & & & 12.7 & 6.0 & 50.2 & 100.0 \\
\hline \multirow{6}{*}{ 70_PTMO2k } & \multirow{6}{*}{70} & \multirow{6}{*}{2} & \multirow{6}{*}{175} & \multirow{6}{*}{$9(35)$} & \multirow{6}{*}{4.5} & 7.3 & 2.6 & 27.2 & 52.9 \\
\hline & & & & & & 8.9 & 3.2 & 33.1 & 66.3 \\
\hline & & & & & & 10.1 & 3.6 & 37.6 & 73.0 \\
\hline & & & & & & 10.3 & 3.7 & 38.5 & 76.0 \\
\hline & & & & & & 13.6 & 4.8 & 50.6 & 100.0 \\
\hline & & & & & & 18.0 & 6.4 & 67.0 & 124.0 \\
\hline 75_PTMO3k & 75 & 3 & 180 & $8.5(29)$ & 5 & 8.6 & 2.5 & 32.3 & 65.6 \\
\hline
\end{tabular}

${ }^{a} \mathrm{M}_{\mathrm{n}}$ of the PTMO diol. ${ }^{\mathrm{b}}$ PBT melting peak maximum extracted from DSC. ${ }^{\mathrm{c}}$ Degree of crystallinity per total sample weight calculated from DSC for the non-SSPCed samples. In brackets, the percentage of crystalline PBT. ${ }^{d}$ Average sequence length of the PBT segments. ${ }^{e}$ Average number of HBs per chain. ${ }^{\dagger}$ Average number of crystallized HBs per chain ${ }^{g} \mathrm{PDI}=M_{w} / M_{n} \sim 2$ for all the samples. $M_{w} \quad$ weight average molecular weight.

$<N>=\frac{M_{n} H B_{w t}}{<L_{n}>M_{0, P B T}}$

Where $M_{0, P B T}=0.22 \mathrm{~kg} / \mathrm{mol}$ is the weight of a single PBT unit. Since not all the HBs in the chain crystallize upon cooling from the melt, we estimate the number of the ones that crystallize as follow:

$<N_{\text {crys }}>=\left\langle N>X_{P B T} / 100\right.$

where $X_{P B T}$ is the percentage of PBT that crystallizes. For clarity, we report the structural parameters in Table 1. From the values of $X$ and the densities of the various phases, $0.98,1.28$, and $1.40 \mathrm{~g} / \mathrm{cm}^{3},{ }^{29,30}$ respectively for amorphous PTHF, amorphous PBT, and crystalline PBT it is possible to estimate the volume percentage of crystallites: $13.7 \%, 7.0 \%$, and $6.5 \%$ respectively for 60_PTMO2k, 70_PTMO2k and 75_PTMO3k.
As not all the HB crystallize, the possibility of glassy HB domains arises; indeed, this is argued in similar systems. ${ }^{19}$ However, previous studies show that for system similar to ours, i.e. with a limited amount of PBT HB, no glassy HB domains are detected. ${ }^{4,5,13}$

\section{Sample preparation}

Granulates are compression molded into either $200 \mu \mathrm{m}$ or $1 \mathrm{~mm}$ thick sheets under vacuum at $230{ }^{\circ} \mathrm{C}$ and allowed to equilibrate for a few minutes at this temperature before being rapidly cooled to room temperature by cooling the press by an internal water circulation system. To define the thickness, Teflon sheets are used as molds, sandwiched between two more Teflon sheets to prevent sticking to the metal plates. The cooling rate was approximately $40{ }^{\circ} \mathrm{C} / \mathrm{min}$, though not constant during the entire cooling time. Due to practical 
equipment issues, some samples are cooled down from the melt at a constant rate of 20 ${ }^{\circ} \mathrm{C} / \mathrm{min}$. To differentiate these samples from the one cooled down with a faster rate, $a * *$ " is placed at the end of their denomination. However, previously collected data (not shown) suggested no appreciable effects of cooling rate on the mechanics at temperatures ranging from room temperature (RT) to $100{ }^{\circ} \mathrm{C}$ for these samples. Example cooling curves are shown in Supplemental Information S1.

\section{Methods}

Tensile bars of ISO 527/5A standard shape are punched out from the $200 \mu \mathrm{m}$ films and tested on a standard Zwick Roell (Germany) 1474-2 Universal tensile testing machine equipped with an air circulation oven for samples at temperatures higher than room temperature and on a Zwick Roell 1455 for the tests at room temperature. Engineering strain is determined using an optical extensometer for increased accuracy, especially in the high strain regime, where strain-hardening and some slippage from the grips are likely to occur. Furthermore, for the tests at room temperature, pneumatic grips are used to further decrease slippage. For all the tests at high temperatures, the samples are equilibrated at target temperature for $10 \mathrm{~min}$ before the test starts. Temperatures are indicated where appropriate in the respective figures and/or captions.

\section{Tensile tests}

During the tensile tests, samples are subsequently stretched at a constant crosshead speed until failure. A preload of $0.05 \mathrm{~N}$ (ca. $0.06 \mathrm{MPa}$ ) is applied before starting. The elastic modulus is calculated at a strain rate of $1 \mathrm{~mm} / \mathrm{min}$ between 0.05 and $1 \%$ of strain. The cross-head speed outside the modulus determination strain is increased to between 5 and $500 \mathrm{~mm} / \mathrm{min}$, corresponding to strain rates, $\dot{\varepsilon}$, ranging from $0.0017 \mathrm{~s}^{-1}$ to $0.17 \mathrm{~s}^{-1}$. The engineering stress and strain curves are plotted to allow for a direct comparison with previous studies on similar materials. Additionally, particularly in the conditions where strain induced crystallization occurs, the assumption of constant volume during deformation might not be correct, which makes it not possible to estimate the true stress.

\section{Cyclic tensile tests}

The cross-head speed for the cyclic tests is set to $50 \mathrm{~mm} / \mathrm{min}\left(0.017 \mathrm{~s}^{-1}\right)$. Deformation steps are indicated in the figures. As in the tensile test, a preload of $0.05 \mathrm{~N}$ is applied before starting. There is no holding time between cycles. Residual strains of each cycle $i$ ( $\left.\varepsilon_{\text {residual }(i)}\right)$ are collected corresponding to the values of strains where the force reached the preload value during unloading. Recovered strain is then calculated by subtracting the applied pre-strain $\left(\varepsilon_{\text {applied(i) }}\right)$ and the residual strain of the respective $i$-th cycle. The tensile set (TS) is also calculated, and is defined as

$T S=\frac{\Delta \varepsilon_{\text {residual }}}{\Delta \varepsilon_{\text {cycle }}}$,

where

$\Delta \varepsilon_{\text {residual }}=\varepsilon_{\text {residual }(i)}-\varepsilon_{\text {residual }(i-1)}$

and

$\Delta \varepsilon_{\text {cycle }}=\varepsilon_{\text {applied }(i)}-\varepsilon_{\text {residual }(i-1)}$.

\section{Differential Scanning Calorimetry (DSC)}

DSC experiments are performed on a TA Instruments (USA) Q2000 instrument. Scans are 
performed at heating rates of $10{ }^{\circ} \mathrm{C} / \mathrm{min}$. Standard $40 \mu \mathrm{L}$ aluminum pans are used and samples weighed $\sim 3 \mathrm{mg}$. Tests are done in air and each pan is punctured prior the test to avoid any pressure build up. Samples are taken from the same films as for the mechanical tests. Additionally, samples are taken near the failure point from RT tensile tested samples to determine if permanent strain induced crystallization (SIC) of the soft blocks occurred. The percentage of crystallized PBT, is calculated from the theoretical enthalpy of fully crystallized PBT $\Delta H_{O}=145.5 \mathrm{~J} / \mathrm{g},{ }^{4}$ as $X_{P B T}=\frac{\Delta H}{\Delta H_{0} * H B_{w t}} \times 100 \%$, while for the degree of crystallinity per total sample weight, $X$, we simply used $X=\frac{\Delta H}{\Delta H_{0}} \times 100 \%$.

\section{Dynamic Mechanical Analysis (DMA)}

DMA tests are performed on an RSA-3 TA Instrument (USA). Rectangular shaped samples (40mm long, $2 \mathrm{~mm}$ wide) punched out from the $200 \mu \mathrm{m}$ hot-pressed films are tested in tension at heating rates of $5{ }^{\circ} \mathrm{C} / \mathrm{min}$ and at a constant frequency of $1 \mathrm{~Hz}$ in the temperature range from $-120{ }^{\circ} \mathrm{C}$ to $\sim \mathrm{T}_{\mathrm{m}}$ in a nitrogen atmosphere. $\mathrm{Tg}$ are collected from the peaks of the $\tan \delta$ curves.

\section{Size Exclusion Chromatography (SEC)}

To determine the $\mathrm{Mw}$, size exclusion chromatography measurements are performed on a Viscotek (UK) GPCMax VE2001 solvent/sample module system, equipped with a TDA302 triple detector array. For chromatographic separation, 3 PFG linear XL columns from PSS Polymer Standards Service $\mathrm{GmbH}$ are used. Detectors and columns are operated at $35^{\circ} \mathrm{C}$. Prior to SEC, the polymer is dissolved at concentrations ranging from 1.0 to
$1.5 \mathrm{mg} / \mathrm{ml}$ in hexafluoroisopropanol containing $0.1 \mathrm{wt} . \%$ potassiumtriflouroacetate (to suppress polyelectrolyte effect since polycondensation can have some localized charge due to tautomeric forms). The molar mass and molar mass distribution is determined with triple detection method, using the refractive index, differential viscosity and right-angle light scattering signals. For calculation of $\mathrm{Mw}$ averages and molar mass distribution, refractive index indices ( $\left.\mathrm{dn} / \mathrm{dc}^{\prime} \mathrm{s}\right)$ in a range of 0.20-0.24 $\mathrm{ml} / \mathrm{g}$ are used. ${ }^{31}$

\section{Atomic Force Microscopy (AFM)}

AFM topology micrographs are obtained using the Peak-Force QNM method on a MM8 from Bruker. Co (USA). Experiments are performed in air, at room temperature, with a frequency around $75 \mathrm{kHz}$, a tip radius of $7 \mathrm{~nm}$ with a nominal spring constant of $7 \mathrm{~N} / \mathrm{m}$. Either the as pressed top surface or cross-section of a $1 \mathrm{~mm}$ sample were imaged. For cross-sections, samples were microtomed at $-120^{\circ} \mathrm{C}$ (below the PTMO glass transition).

\section{X-ray analysis (SAXS/WAXS)}

The experiments are carried out on a GANESHA 300XL (SAXSLAB, USA) + system from JJ X-ray (Denmark). The instrument is equipped with a Pilatus $300 \mathrm{~K}$ (DECTRIS, Switzerland) detector, with a pixel size of $172 \mu \mathrm{m} \times 172 \mu \mathrm{m}$. The X-ray source is a Genix 3D (Xenoxs, France) Microfocus Sealed Tube X-Ray Cu-source with integrated Monochromator (multilayeroptic "3D version" optimized for SAXS) (30 W). Calibration of scattering angle is done using silver behenate $\left(d \_001=58.380 \AA\right.$ ). Samples are fixed in two oppositely moving clamps, so that the acquisition point stays constant for measurement. Step-loading is applied here to provide sufficient time for the X-ray data 
collection period. The cross-head speed is 20 $\mu \mathrm{m} / \mathrm{s}\left(0.002 \mathrm{~s}^{-1}\right)$ and measurements were performed at engineering strains (determined from crosshead displacement) of $0 \%, 100 \%$, $300 \%, 500 \%$ and $700 \%$. Measurement begins 5 min after reaching the target strain to avoid a strong influence from relaxation directly after loading. The measurement period is $15 \mathrm{~min}$ for WAXS and $30 \mathrm{~min}$ for SAXS. The X-ray wavelength is $0.124 \mathrm{~nm}$ and the switch between WAXS and SAXS at each strain is achieved by the translation of the detector. The distance from the sample to the detector for SAXS and WAXS is $415 \mathrm{~mm}$ and $120 \mathrm{~mm}$, respectively. A picture of the experimental set-up is shown in Supplemental Information S2. Analyzing the curves by means of fitting to obtain details on the morphology is beyond the scope of this study. Additionally, the weakness of the X-ray signal coming from the low crystallinity and thickness of the samples, as well as the broadness of the peaks coming from the polydispersity of the $\mathrm{HB}$, make the identification of the peaks difficult and the uniqueness of the fit problematic.

\section{RESULTS}

\section{Morphology}

Modulus mapping via the AFM QNM method of the surface (Figure 1) and cross-section (Supplemental information S3) of hot-pressed samples at ambient conditions are consistent in showing the presence of ribbon-like crystals with high aspect ratio in a softer matrix. In both the figures no spherulites are detected at the investigated length scales. The crystals are stacked PBT segments while the softer matrix is a mixture of PTMO + un-crystallized PBT. For soft systems (HBwt\% < 50), crystals do not form spherulites as in harder systems. ${ }^{14}$ Furthermore, the low HBwt\% content results in shorter HBs on average (lower $\left\langle L_{n}\right\rangle$ ) which makes chain folding during crystallization of the PBT unlikely to occur. The crystals are connected via soft segments (amorphous SB and un-crystallized $\mathrm{HB}$ ) that can bridge crystallized segments in the same or different crystals and can form trapped entanglements.

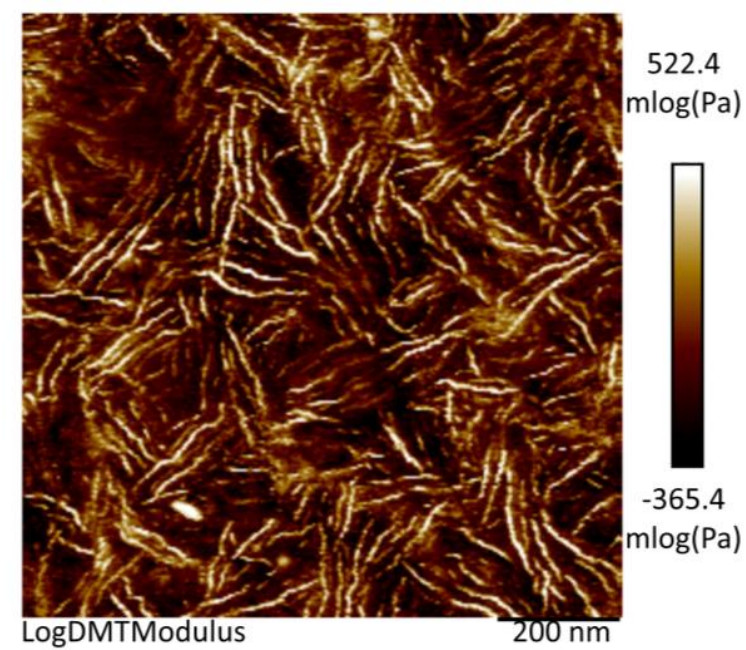

FIGURE 1 AFM micrograph (QNM) showing the top surface of a hot-pressed 70_PTMO2k_33 sample at ambient conditions. Brighter regions correspond to a higher elastic modulus (PBT crystals) while darker regions correspond to the PTMO-rich soft phase.

The molecular weight between entanglements $\left(M_{e}\right)$ of PTMO is $\sim 1.4 \mathrm{~kg} / \mathrm{mol}^{32}$ which makes the amorphous chain segments between crystals well entangled. Despite the absence of a cocontinuous hard phase, the high aspect ratio of the ribbon-like crystals shown in the AFM micrographs introduces the possibility of a mechanically-interlocked network of crystals contributing to the initial stiffness of the material, which will be discussed later on in the paper. In Supplemental Information S4 we provide SAXS signals for the sample in the undeformed state. 


\section{Thermal transitions}

The tensile storage moduli ( $\left.E^{\prime}\right)$ in the linear range versus temperature for different $\mathrm{SB} / \mathrm{HB}$ ratios and $\mathrm{Mw}$ are shown in Figure 2 . The general trends are similar for all the materials. DSC traces are shown in Figure 3 (cooling curves in Supplemental Information S6). The PBT crystal $T_{m}$ is determined by the crystal size and perfection and increases with the average block length $\left.\left(<L_{n}\right\rangle\right){ }^{4}$ Thermal transitions observed via DSC and DMTA yield a consistent picture (note that the measurements are performed as slightly different heating rates). We will next describe briefly the major transitions observed with increasing temperature based on these results as well as on the extensive previous literature. ${ }^{1,3,4,13}$

At around $\sim-60^{\circ} \mathrm{C}$ the $\mathrm{T}_{\mathrm{g}}$ of the PTMO-rich phase is observed which for high amounts of high-Mw SBs is relatively independent of the soft block content. ${ }^{13}$

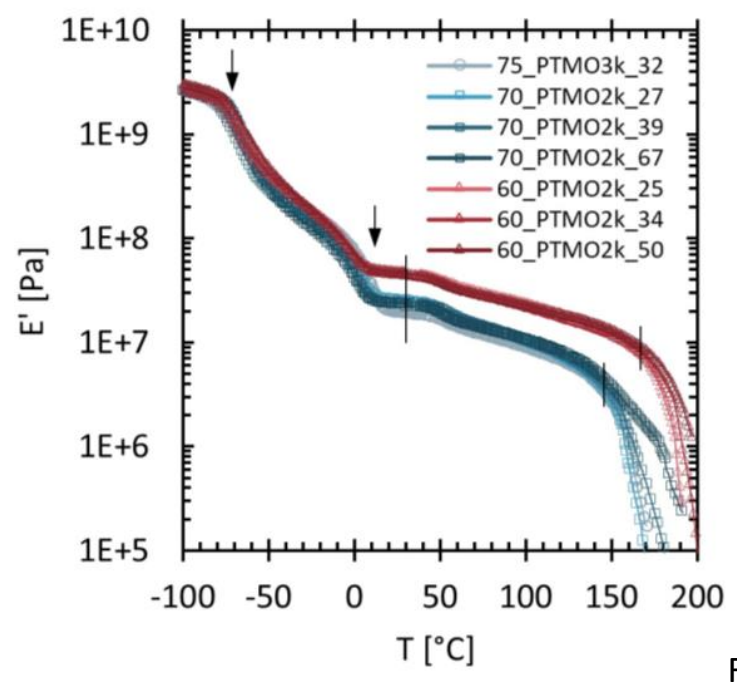

$\mathrm{FI}$

GURE 2 Storage modulus for the series of different $M_{n}$ of samples 60_PTMO2k, 70_PTMO2k and 75_PTMO3k. $M_{n}$ increases from light to dark. The arrows indicate the $T_{g}$ and the end of melting for the PTMO, the vertical lines indicate the start and end off the temperature range where nonlinear properties were studied. Loss modulus and $\tan \delta$ related to the same set of samples are shown in Supplemental Information S5.

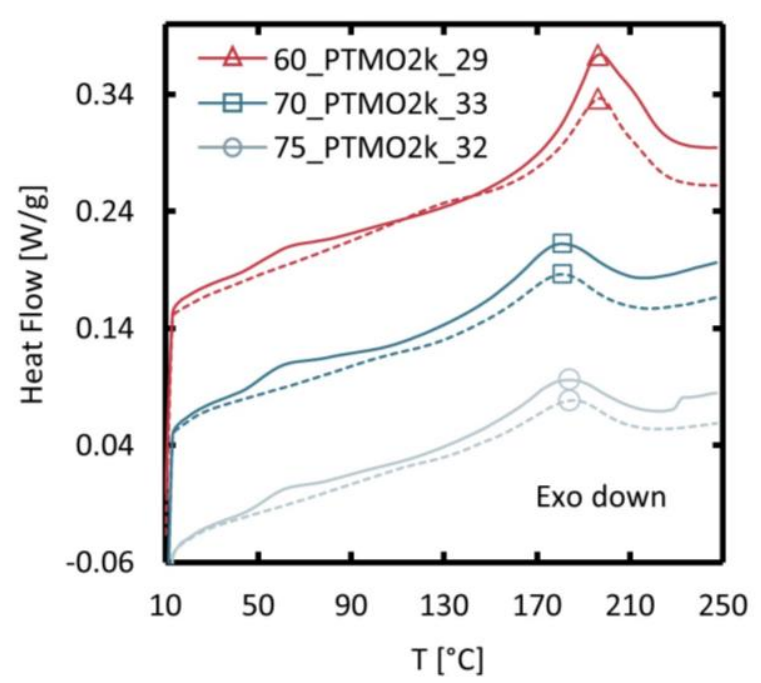

FIGURE 3 DSC thermographs from $10{ }^{\circ} \mathrm{C}$ to 250 ${ }^{\circ} \mathrm{C}$ for three samples. The continuous lines correspond to the first heating, the dashed ones to the second heating. Symbols indicate the respective peak maxima. Data are vertically shifted.

Next the melting of PTMO is observed around $\sim 0-10{ }^{\circ} \mathrm{C}$, the melting point increases with the PTMO length and the level of crystallinity increases with the amount of PTMO. ${ }^{12}$ At higher temperatures, the system consists of crystallized PBT segments in a mobile ( $T$ > $T_{g}$ ) amorphous matrix, and a rubbery plateau is observed. In this region, the elastic modulus mainly scales with crystallinity, ${ }^{19}$ which increases with the amount of $\mathrm{HBs}$ in the system. ${ }^{2}$ The end of the rubbery plateau is dictated by the melting of the PBT-crystals. Unlike a traditional cross-linked rubber, the modulus decreases in the plateau region with increasing temperature. This is attributed to the increased mobility in the amorphous phase surrounding the PBT crystals. Indeed, due to the polydispersity of the HB length, the interface 
between the crystalline and amorphous phases is not expected to be well defined. A PBT-rich mixed phase is likely to be at this interface, whose increased mobility with increasing temperature can contribute to the $\mathrm{E}^{\prime}$ decrease.
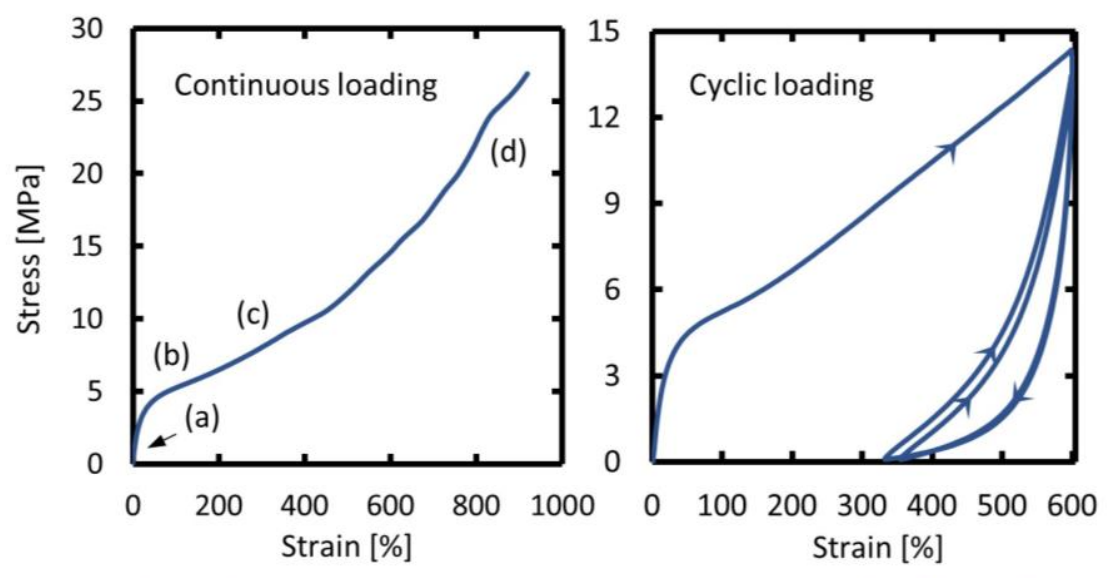

(a)

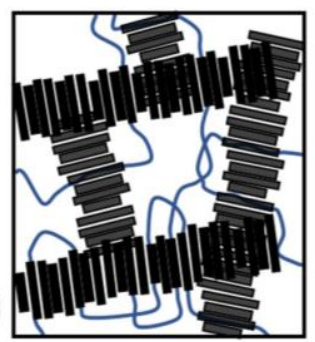

(b)

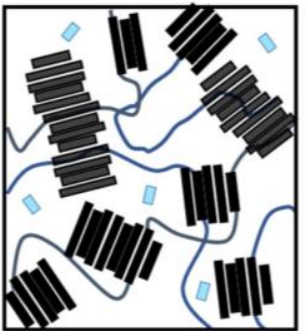

(c)

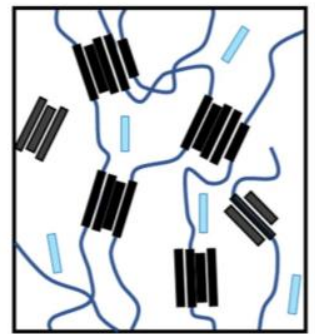

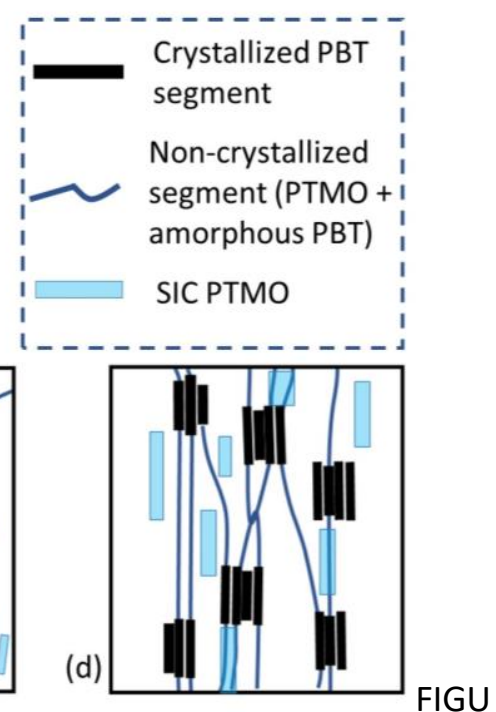

RE 4 Top-left) Example of an engineering tensile curve, letters in the plot indicate roughly the points on the curve where the morphology is expected to be different, referring to the ones in the bottom picture. Top-right) Plot showing the response of cyclically loading the material several times to the same strain resulting in the decrease in modulus and hysteresis between the first and following cycles. Arrow indicate loading and unloading. Bottom) Schematic cartoon representing the evolution of the morphology upon stretching: deformation causes the crystalline stress-bearing structure (a) to break and the material to progressively transit to an elastomeric-like structure (b-c), where the broken crystal domains progressively reorient towards stress direction and act as physical crosslinking points. Further stretching leads to chain alignment and pull-out of some of the crystallized PBT segments from the crosslinks. The competition between the latter and the stretching of the chain segments is responsible for the stress upturn. Eventually, stretching leads to a very aligned morphology (d) where, if temperature allows SIC of the PTMO segments dominates the mechanical behavior.

Note that as the material nears the melting point of the PBT, the decrease is more rapid. This is likely due to the melting of the less perfect crystals or of the crystals formed by shorter HBs, rather than to the increased mobility of the phase surrounding the crystals. For an extended discussion on this topic see De
Almeida et al. ${ }^{6}$ Figure 2 shows also that the $\mathrm{Mw}$ of the chains does not affect the linear modulus in the plateau region, as would be expected if the amount of crystallinity and the initial crystal morphology were not affected by Mw. 
Influence of deformation on morphology and modulus.

To further understand how deformation influences the morphology and mechanical properties of these materials cyclic loading experiments were performed. As an example, a cyclic test where the material is loaded and unloaded three times up to the same target strain is shown in Figure 4 (top-right) illustrating the different mechanical response between the first and second cycles. Actual cyclic tests are shown in Supplemental information (S7). Several key features should be noted: after initial deformation, the material shows a large amount of elastic recovery and, on reloading, the modulus of the deformed material is significantly lower than for the undeformed material. As shown in Figure 5, the elastic modulus of the reloaded sample initially decreases with applied pre-strain. Due to strain the material acts as if it was transitioning from a response dominated by an original, mechanically interlocking and stress-bearing PBT-crystal network to a more elastomeric one where the crystals act as isolated cross-linking points. This rapid drop in modulus with strain has been shown for other multi-block copolymers with a similar strain-evolving structure. ${ }^{33-35}$ Finally, after experiencing very high strains, a highly oriented system with both small PBT crystals and with amorphous segments aligned along the stretching direction (and possibly crystallized) is obtained. The morphology evolution with applied strain is illustrated schematically in Figure 4. There is not yet consensus on what determines the initial modulus for soft TPEs, how it increases with HB content, and why it is strongly reduced after strain. Based on data from several chemically different TPEs with crystal volume fraction < $40 \%$, Nebouy et al. $^{19}$ argue the crystallites structure, in particular the volume fraction and the width of the crystals, determines the linear modulus.

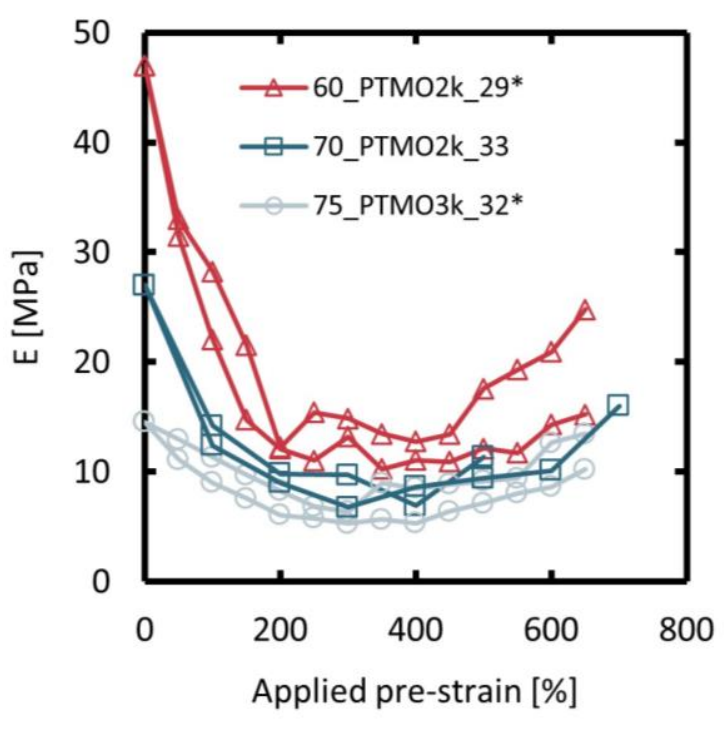

IGURE 5 Young's modulus (E) versus applied pre-strain. Data calculated from cyclic experiments (Supplemental Information S7 and S8) performed at room temperature. Duplicates are shown. Values are corrected for the change in cross-section during stretching, assuming constant volume.

Their theoretical arguments takes into account the covalent bonds between the soft and hard segments and local topological arguments by treating the crystals as local densifications of the polymer network. ${ }^{36}$ In such a model, the reduction in modulus with strain requires a significant reduction in associated $\mathrm{HBs}$ or in crystal width. An alternate approach is to treat the HB domains as rigid, dispersed fillers in analogy with standard composite theory. ${ }^{10}$ We interpret the as molded sample's stiffness as dominated by the rigid crystal phase which carries the load, in analogy with a foam type structure. As such, the modulus would be expected to scale with the volume fraction of crystallinity as well as the bending stiffness of 
the crystals. ${ }^{37}$ The rigidity of this stiff network greatly exceeds that of the entangled amorphous phase connecting the crystals and dominates the initial response. During strain, the crystals undergo yielding to break into smaller segments ultimately resulting in dispersed crystals and in the stiffness being dominated by the amorphous phase. This transition from stiffness being dominated by a mechanically interlocking crystal network to that of the entanglement amorphous phase explains the large drop in modulus and hysteresis at modest strains $(<200 \%)$ without involving a large drop in associated hard blocks. At the extreme where the $H B$ fraction is sufficiently low, a rigid network should not be able to form and the initial modulus and hysteresis should be low. What is surprising is that even for these systems with relatively low amounts of crystallinity the same phenomenological response is observed. Studies that monitor the change in associated $\mathrm{HB}$ with deformation would be extremely useful in further elucidating which conceptual model for understanding the modulus in soft TPEs is correct.

After large deformation we see an increase in modulus. We believe this can be attributed to the high level of alignment of the chains and also a contribution coming from SIC of the PTMO. DSC performed on broken specimens after tensile tests show a new endo-thermic peak between 40 and $50{ }^{\circ} \mathrm{C}$, corresponding to SIC of the PTMO (Supplemental Information S6). ${ }^{8}$ This is also supported by the in-situ X-ray tests (Supplemental Information S9) which show the PTMO crystals are present both during applied strain and after unloading, i.e. the so formed crystals are stable even after unloading and disappear only after the temperature is raised. Figure S9(a) shows that
PTMO crystallinity progressively increases with applied strain and already starts forming before the strain hardening onset.

\section{Nonlinear properties}

Now that we have established a morphological picture for these systems and how it changes upon loading, we switch to investigating how composition, $\mathrm{Mw}$ and temperature affect the mechanical performance.

Figure 6 shows engineering stress-strain curves at RT, $100{ }^{\circ} \mathrm{C}$, and $150{ }^{\circ} \mathrm{C}$ for difference $\mathrm{HB} / \mathrm{SB}$ ratios (a-c) and total chain $M_{n}(\mathrm{~d}-\mathrm{f})$.

\section{Varying SB/HB ratio - fixed Mw}

We will begin by examining the influence of $\mathrm{SB} / \mathrm{HB}$ ratio at similar total $\mathrm{Mw}\left(M_{n} \sim 30 \mathrm{~kg} / \mathrm{mol}\right.$, PDI 2) as depicted in Figure 6(a-c). The general features are similar for all curves: the stress increases roughly linearly to $10 \%$ strain. Then, the slope begins to decrease which we attribute to plastic deformation (yielding) of the crystalline domains of PBT. Next, the stress mildly increases to $\sim 300 \%$ strain when the material starts to harden. Finally, high stresses and strains are reached resulting in breakage.

All samples show a decrease in failure stress and strain with increasing temperature, but the magnitude of the temperature effect depends on the amount of soft block in the system. It is observed that increasing $\mathrm{SB}_{\mathrm{wt}}$ leads to a reduction in temperature resistance. The materials are compared at very similar $\mathrm{Mw}$ but have different relative distances of the testing temperature from the $T_{m}$. Figure 7 better summarizes the effects of the temperature on the failure strains as the SB/HB ratio changes, by showing the failure strains collected from tensile tests versus the distance from the melting peak temperature $\left(T_{m}-T\right)$. 

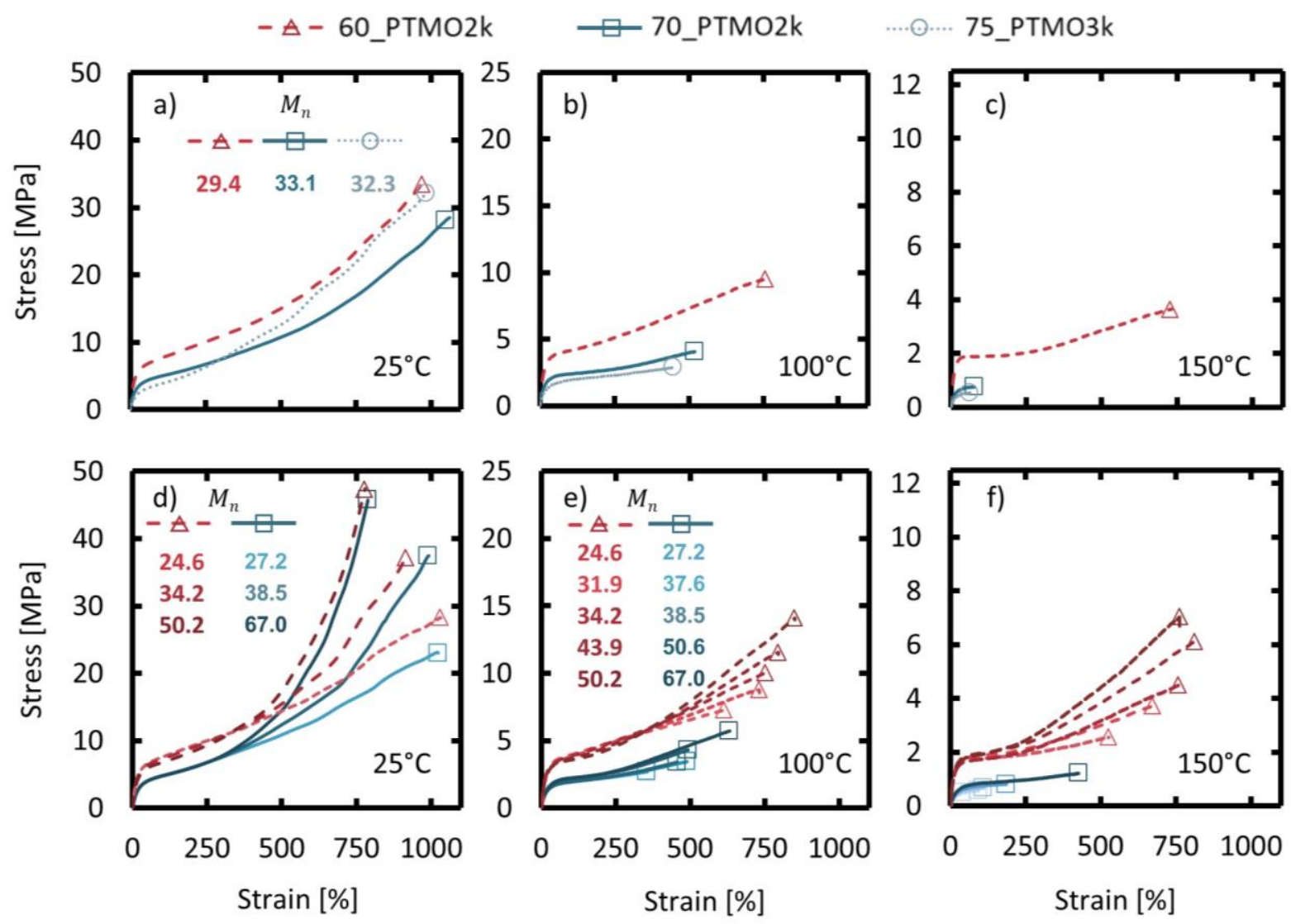

URE 6 Representative engineering stress-strain curves for a-c) 60_PTMO2k_29, 70_PTMO2k_33 and 75_PTMO_32 and for $d-f$ ) the Mw series of the samples 60_PTMO2k and 70_PTMO2k, at RT (for a and d), $100{ }^{\circ} \mathrm{C}$ (for $\mathrm{b}$ and e) or $150{ }^{\circ} \mathrm{C}$ (for $\mathrm{c}$ and f). All the tests are conducted at fixed strain-rate $\dot{\varepsilon}=0.17 \mathrm{~s}^{-1}$. The $\mathrm{Mw}$ is approximatively the same $\left(M_{n} \sim 30 \mathrm{~kg} / \mathrm{mol}\right)$ for all the materials in a-c). In d-f) $M_{n}$ increases from light to dark. Open symbols indicate the failure stress and strain of each sample.

Note that 75_PTMO3k has a higher melting temperature compared with 70_PTMO2k (due to the longer PTMO diols resulting in longer PBT segments) but shows less temperature resistance.

This implies that the relative distance from $T_{m}$ is not enough to explain the temperature resistance of these systems. If the temperature resistance is related to connectivity then it would be expected to increase with the crystallinity and the number of crystallized HB per chain, $\left\langle N_{\text {crys }}\right\rangle$. This is consistent with the behavior observed for these systems at high temperature (see Table 1).

At RT the strain-hardening is much stronger than at higher temperatures for all materials, and it is worth noting that the ultimate failure stress at room temperature does not scale with the linear modulus. If PTMO blocks are sufficiently long, they can crystallize under strain resulting in a shift in melting temperature from $\sim 0{ }^{\circ} \mathrm{C}$ to $\sim 50{ }^{\circ} \mathrm{C} .8,38$ Previous studies ${ }^{13,38}$ 
showed that the amount of SIC increases with the amount and length of soft blocks. Furthermore, the PTMO length also influences the value of strain at which we start to see $\mathrm{SIC}^{13}$ the longer the SB length, the earlier the onset of SIC. This is in line with our observation from the tensile tests at RT where the 75_PTMO3k shows the highest strain-hardening among the tested grades.

At $\mathrm{T}>50{ }^{\circ} \mathrm{C}$ the strain-hardening of the 75_PTMO3k is comparable to the other grades. This underscores the importance for morphology evolution including contribution of SIC on the non-linear mechanical response.

\section{Varying $M w$ - fixed SB/HB ratio}

Turning now to systems of fixed SB/HB ratio but varied $\mathrm{Mw}$, in Figure 6(d-f) we see first that the $\mathrm{Mw}$ has little influence on the behavior up to the strain at which strain-hardening starts ( $300 \%)$. Again, this is consistent with the first effect of strain being to break-up the crystal network, whose structure and properties are insensitive to total chain length. At higher strains increased $\mathrm{Mw}$ leads to increased strain hardening and, at high temperatures, also to an increased extensibility. As mentioned previously, at RT the strain-hardening is mainly determined by PTMO SIC. Despite having the same amount of SB and same SB length, the high-Mw samples show a considerably higher strain-hardening, which suggests that higher Mw leads to a more effective SIC.

This is supported by the in-situ X-ray, where the high-Mw sample orients sooner and to a higher extent than the low-Mw one (Supplemental Information S10and S11).

At temperatures higher than the melting of the PTMO SIC $\left(\sim 50^{\circ} \mathrm{C}\right)$, increasing the $\mathrm{Mw}$ still leads to a considerable increase of the strainhardening. Therefore, there must be an additional mechanism in addition to SIC that is responsible for the change in high strain behavior.

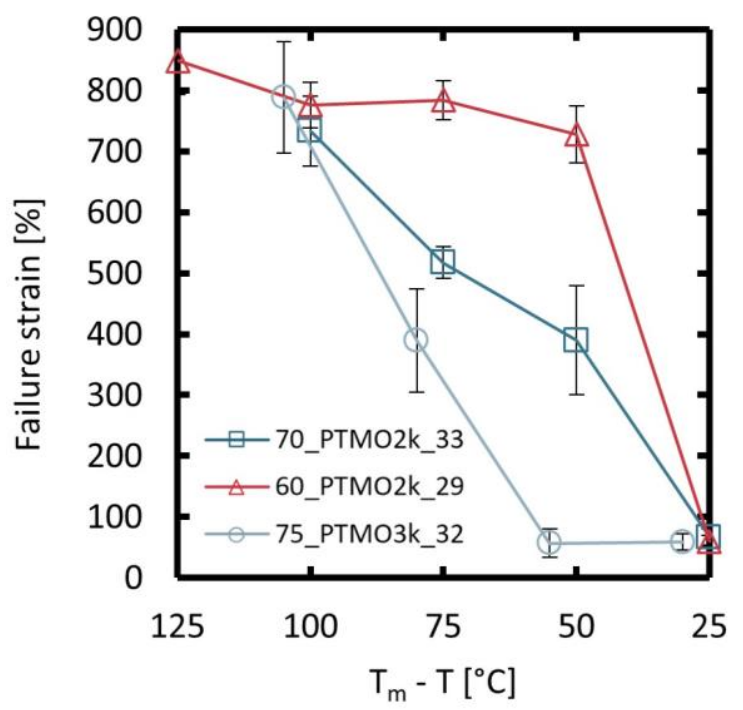

FIGURE 7 Failure strain versus the difference between the melting peak temperature and the testing temperature $\left(T_{m}-T\right)$. Failure strains are taken from tests shown in Figure 6 as well as from additional tests. $T_{m}$ are determined by DSC and given in Table 1 and Figure 3. Note data < $75^{\circ} \mathrm{C}$ is excluded to avoid influence of SIC.

Additional insights into the influence of composition and $\mathrm{Mw}$ on the morphology and the mechanics can be gained from cyclic tests (cyclic curves in Supplemental Information S7). Figures 8(a) and 8(b) show the recovered elastic strain at each step of deformation for the 70_PTMO2k samples with two different $\mathrm{Mw}$ at $\mathrm{RT}$ and $100{ }^{\circ} \mathrm{C}$. (Data relative to the samples 60_PTMO2k_29* and 60_PTMO2k_50* are shown in Supplemental Information S12). At each step strain, the high-Mw sample recovers more strain, i.e. the residual strain at zero force is lower compared to the low-Mw counterpart. The Mw dependence of the recovered strain is stronger at higher temperatures, probably due 
to the increased chain mobility and lack of

b)

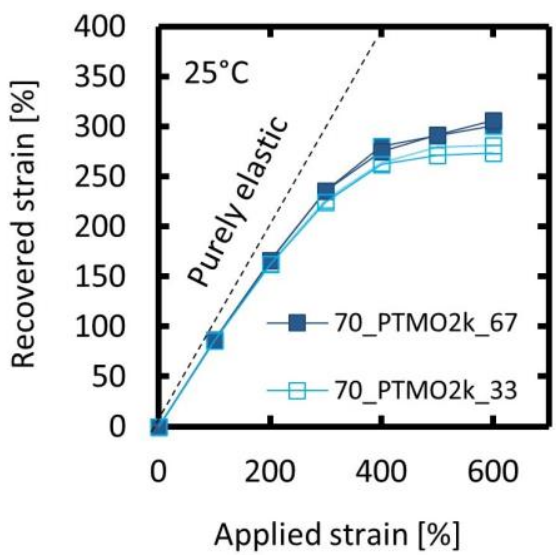

a)

Applied strain [\%]
PTMO

SIC.

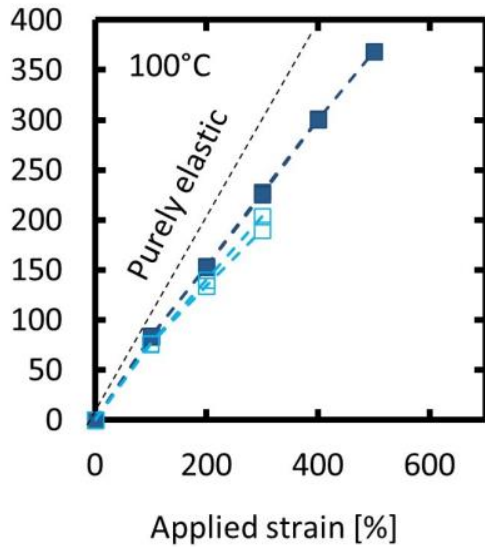

c)

FIGURE 8 a) Plots showing recovered strain obtained from cyclic tests performed respectively at a) RT and b) $100{ }^{\circ} \mathrm{C}$. The dashed line indicates the purely elastic behavior. c) Tensile set (TS) versus the applied pre-strain at both RT (continuous lines) and $100{ }^{\circ} \mathrm{C}$ (dashed lines). Duplicates are shown.

The SIC increases the plastic contribution upon stretching as illustrated by the large deviation from the purely elastic response at higher strains. The applied pre-strains are the same at each cycle for the two materials, but the recovered strains are different, i.e. the high-Mw sample recovers more strain. As a consequence, at each cycle the high-Mw sample is stretched more than the low-Mw one. This effect is taken into account in Figure 8 (c) by plotting the tensile set (TS) for the same materials against the applied pre-strain. From the definition of tensile set in the Method section, the higher TS, the higher the plasticity in the material. The data show dramatically different behavior at RT and at $100{ }^{\circ} \mathrm{C}$. Indeed, the $T S$ increases with deformation at RT, while it decreases at $100{ }^{\circ} \mathrm{C}$. Additionally, the differences in TS between the two $\mathrm{Mw}$ samples are higher at $100{ }^{\circ} \mathrm{C}$, where SIC does not overshadow the elastic contribution coming from the network connectivity. This confirms the stronger network elasticity present in the high-Mw sample, that we attribute to an increased network connectivity with $\mathrm{Mw}$, attributed to the lower amount of dangling-ends and increased amount of HB per chains, as described quantitatively and more in detail in the last section of this paper. This supports the idea that upon stretching the morphology gradually evolves towards an elastomeric-like one (in the case of no SIC).

Interestingly, the tensile set analysis reveals differences with $\mathrm{Mw}$ already at low strains, which is different from the identical behavior in the continuous loading tensile tests in Figure 6. In fact, during loading, the plastic contribution coming from the yielding of the crystals is what contributes most to the stress response and overshadows the contribution coming from the network connectivity. Unloading is largely an elastic response and, hence, more sensitive to the influence of pre-strain on the network connectivity. Figure 2 and Figure $6(d-f)$ show that there are no differences with $\mathrm{Mw}$ on the loading curves before the strain-hardening onset, suggesting that the initial crystalline network is the same for samples with different Mw. 

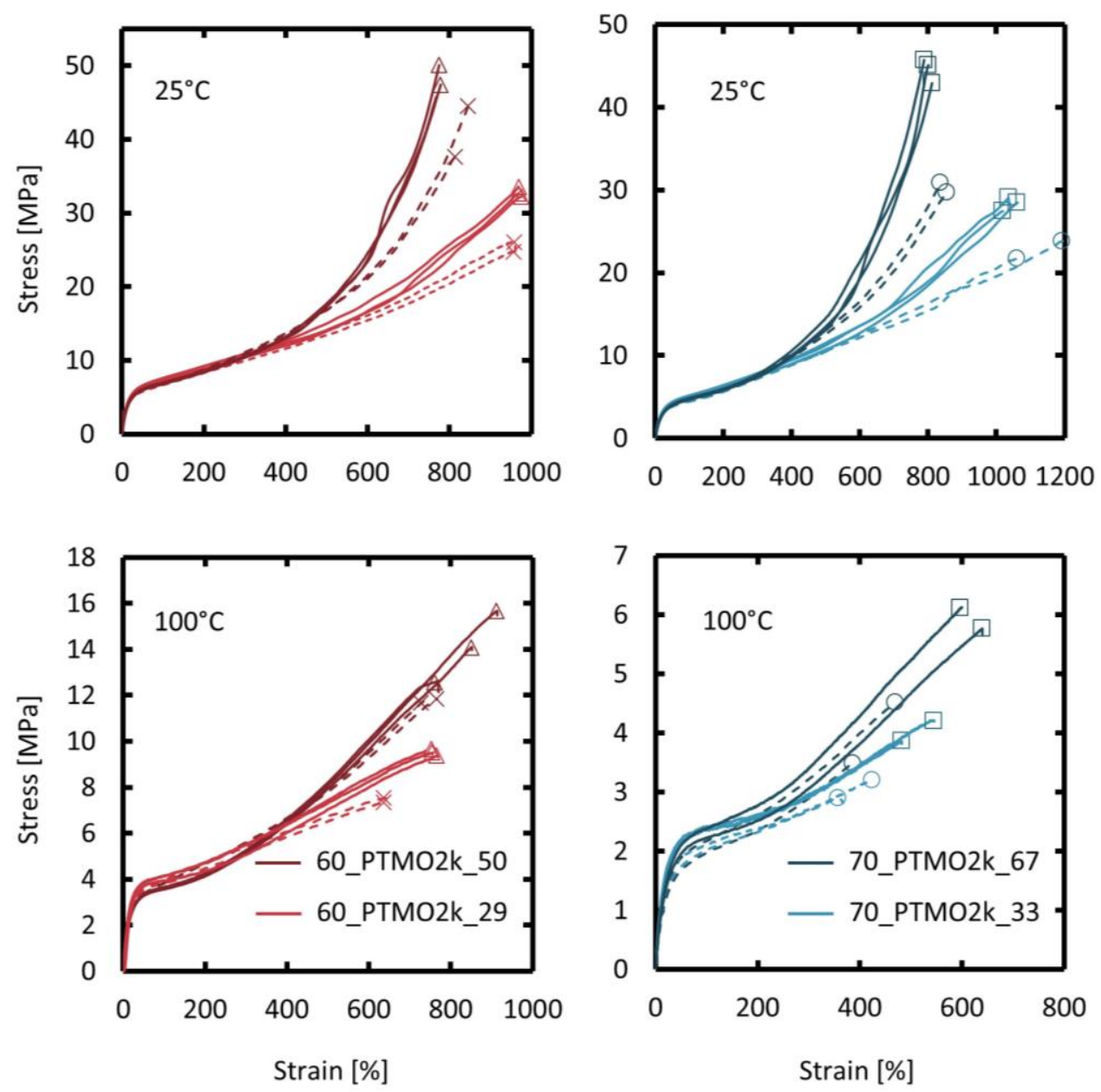

FIGURE 9 Engineering stress-strain curves at RT and at $100{ }^{\circ} \mathrm{C}$ at strain-rates of $0.17 \mathrm{~s}^{-1}$ (continuous lines) and $0.0017 \mathrm{~s}^{-1}$ (dashed lines). Duplicates are shown.

In contrast, the unloading analysis in Figure 8(c) clearly shows increased elasticity with $\mathrm{Mw}$ (e.g. lower $T S$ at $100^{\circ} \mathrm{C}$ for the higher $\mathrm{Mw}$ ). It is also worth noting that while the modulus drops strongly at strains $<200 \%$, the $T S$ and the recovered strains are less influenced at this low to moderate strain level.

\section{Strain-rate effects.}

Finally, we show how the strain-rate dependence changes with Mw. Figure 9 shows the engineering stress-strain curves at RT $100{ }^{\circ} \mathrm{C}$ for 60_PTMO2k and 70_PTMO2k, at two different strain rates and $\mathrm{Mw}$.

At RT, decreasing the strain-rate leads to a decrease in strain hardening and to an increase in final extensibility. This is possibly due to a lower amount of PTMO SIC at low loading rates. At $100{ }^{\circ} \mathrm{C}$, decreasing the strain-rate leads to a decrease in the extensibility as was observed by Aime et al. ${ }^{27}$ for very similar systems. This is attributed to a stress-accelerated dissociation of $\mathrm{HBs}$, i.e. Eyring-like kinetics resulting in a creep-like failure mechanism. Similar to what is 
theorized for many other thermal activated processes over an energy barrier that decreases with applied. ${ }^{39-41}$

This behavior does not seem to be $\mathrm{Mw}$ or composition dependent. More interestingly, we observe a different behavior with $\mathrm{Mw}$ concerning the strain-hardening dependence with the strain-rate: the strain-hardening generally decreases as the strain-rate decreases. The effect on stress is much less pronounced for the high-Mw samples although rate still affects failure strain. To the best of our knowledge, this difference strain rate response for different $\mathrm{Mw}$ has not been reported before. The lower strain-rate dependence at high $\mathrm{T}$ for the high-Mw samples in combination with the increased elasticity and strain-hardening with $\mathrm{Mw}$ supports the idea of a relaxation mechanism for the stress bearing units in the system that is temperature- and $\mathrm{Mw}$ dependent. The hypothesis of temperature and strain-rate dependent kinetics of disentanglement has been carried out for linear monodisperse PE both in experiments ${ }^{42}$ and simulations. $^{43}$ The observations have been attributed to entanglements being the fundamental stress bearing units in the system at large deformation. In our case, the presence and associations of the HBs must be taken into account as they have their own stress-, temperature- and time-dependent kinetics. ${ }^{27}$ This will presumably also affect the entanglements dynamics since they prevent chain diffusion and disentanglement.

In the following section we start from the experimental observation to build a physical picture for the evolution of the microstructure upon deformation, highlighting the different dynamics responsible for the mechanical behavior and providing a qualitative physical model that reasonably explains the results obtained from the tensile and cyclic tests.

\section{DISCUSSION}

\section{Morphology evolution}

Our physical picture for the morphology evolution is largely based on analyses in previous studies on similar materials. ${ }^{1,13,25}$ The microstructural evolution with strain is depicted in Figure 4. The mechanical properties in the linear regime and up to the yield region are mainly governed by the crystallinity of the system. The linear modulus is dominated by the volume fraction of ribbon-like PBT crystals with little influence of total chain length. With increasing deformation, the morphology evolves via crystal breaking and orientation, as indicated by the decrease in the crystal size with deformation and by the long period which increases at small deformation and decreases at strains $>\sim 100 \%$ as a result of the breaking mechanism. ${ }^{1,20,21}$ Our results from SAXS (Supplemental Information S11(b)) also confirm the decrease in long period with deformation. The broken crystalline domains re-orient such that the PBT and the PTMO segments are aligned along the stress direction. Up to $\sim 300 \%$ strain, the Mw of the total chain does not affect the response in a continuous loading experiment since it does not significantly influence the crystallinity or initial morphology. However, unloading analysis reveals differences with increasing $\mathrm{Mw}$ increasing the elastic recovery. By $\sim 300 \%$ strain the break-up and reorientation process of the PBT crystals is mostly complete, but the crystal disruption and fragmentation will continue with increased strain due to the pull-out of the PBT segments from the crystals. ${ }^{27}$ At higher strains, a strong Mw-dependence on strain-hardening is observed. Thus, starting from a rigid 
mechanically interlocking network of crystal ribbons, the morphology evolves with strain into a network with fragmented PBT crystals acting as isolated physical crosslinks. Eventually to a highly oriented structure where, if temperature allows, SIC of the SBs can additionally contribute to the stress response.

\section{Strain-hardening and network elasticity}

The nature of strain-hardening is still a topic of debate for semi-crystalline polymers and only a few observations where molecular parameters are varied in a systematic fashion are present in literature, with especially limited observations at high temperatures.

Kennedy et al. ${ }^{44}$ hypothesized that the strainhardening increase with $\mathrm{Mw}$ in semi-crystalline random copolymers of ethylene, which behave very similarly to these TPE-Es, ${ }^{45}$ can be explained either by SIC of the stretched copolymer and increased elasticity in the amorphous disordered inter-crystalline region due to the orientation and stretching of the entangled segments.

In our case, the first mechanism is dominant at $\mathrm{T}<\sim 50{ }^{\circ} \mathrm{C}$ due to PTMO SIC. Increasing the $\mathrm{Mw}$ leads to an increased connectivity which results in a better, more efficient orientation and more persistent network during stretching. This amplifies the effect of the PTMO SIC on the mechanics (Supplemental Information S10). However, at higher temperatures, the hardening response should come entirely from the increased elasticity in the amorphous intercrystalline region, which is consistent with the results obtained from the cyclic tests (Figure 8).

For semi-crystalline homopolymers it has been observed $^{46}$ that an increase in $\mathrm{Mw}$ strongly increases the strain-hardening while decreasing the drawability and the stresses at failure. This effect has been correlated with the amount of tie molecules and especially entanglements in the amorphous region between crystals. ${ }^{47} \mathrm{~A}$ review on the argument has been published by Bartczak. ${ }^{48}$ Kennedy et al. presented results for semi-crystalline polymers showing that increasing $\mathrm{Mw}$ leads to an increase of the entanglement density in the amorphous region ${ }^{46}$ due to the expulsion of entanglements from the crystalline phase during cooling from the melt. This results in an earlier onset and stronger strain-hardening response. Since the crystals in our system are obtained via PBTsegments stacking and not via folding (the PBT segments are too short to allow even for a single fold), we assume that the entanglement density does not significantly change from that of the melt when the $\mathrm{Mw}$ is varied. Furthermore, we do not observe a significant shift in the on-set of strain-hardening. In summary, the previous findings on the nature of strain-hardening based on the study of semicrystalline homopolymers do not fully explain the results our TPE-Es.

In the following section we propose a phenomenological model for the increased strain-hardening, elasticity and ductility observed at high temperature $\left(\mathrm{T}>\sim 50{ }^{\circ} \mathrm{C}\right)$ for the high-Mw samples, based on the connectivity of both PBT crystals and the entangled network.

\section{Residual connectivity and Stress Bearing Units (SBUs).}

The model aims to count the number of stress bearing units (SBUs) in the system at each stage of deformation, meaning that the non-relaxed segments between two entanglements and between entanglements and PBT-crystals are both counted. The goal is to propose a 
qualitative mechanism for the stress response which is time- and temperature- dependent, with this dependency decreasing as $\mathrm{Mw}$ increases. During deformation, due to the pullout of the crystalline PBT segments from the respective crystals, the number of crosslink points and therefore, the overall connectivity of the system decreases. ${ }^{27}$

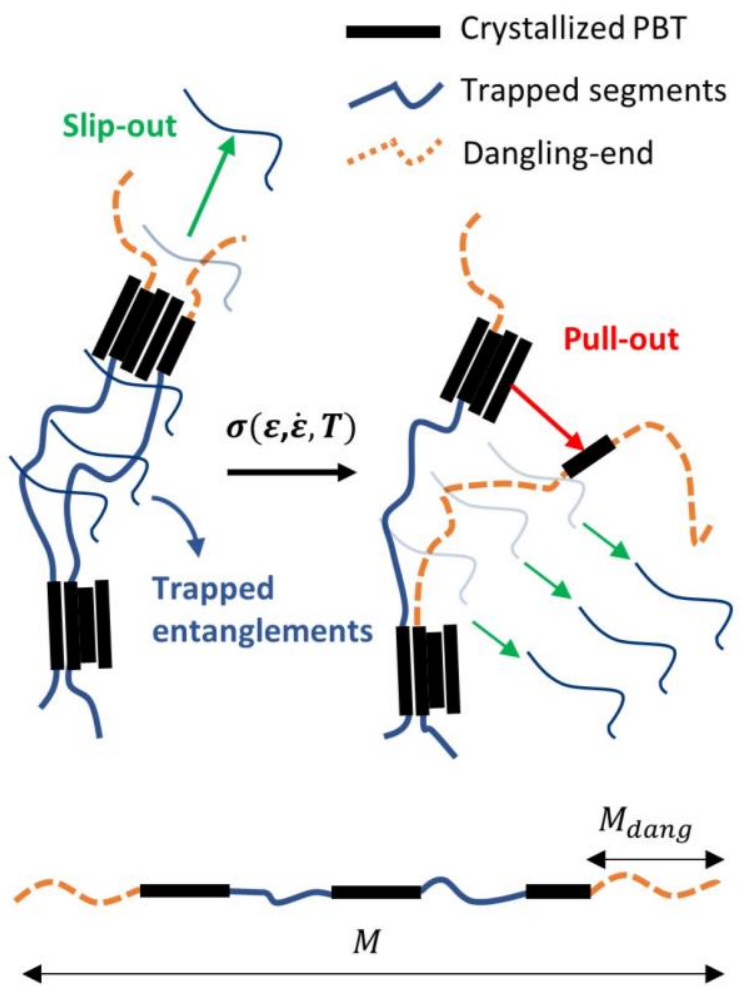

FIGURE 10 Cartoon representing the idea of the disentanglement allowed (dashed to solid lines) only for the segments of chains not trapped between two crystallized HB. Dangling ends can slip-out from entanglements. As pull-out events occur, segments of chain are not trapped anymore and can now escape the entanglements. At the same time, the length of the dangling-ends increases, increasing the time needed to escape.

We assume that the pulled out PBT segments do not associate again. In this framework, the crystallized $\mathrm{HB}$ contribute to the stress response by keeping segments of chains trapped and ensuring connectivity. For simplicity, all the chains are treated as monodisperse in total chain length $(M)$ and block lengths and the focus is on the microstructure where the initial crystal network has broken up (Figure 4(c)) and the temperatures are above the melting of the SIC. Since the emphasis is mainly on the effect that the Mw has on the SBUs at different strain rates, the figures below focus on the analysis done on a single system, i.e. the 70_PTMO2k series, with fixed amount of SB (SB wt $\left._{w}=70 \%\right)$, SB length $\left(\mathrm{M}_{\mathrm{n}, \mathrm{SB}}=2 \mathrm{~kg} / \mathrm{mol}\right)$ and crystallinity $\left(X_{P B T}=\right.$ $35 \%)$.

Since the testing temperatures are much higher than the $T_{g}$ of the soft PTMO-rich phase $\left(T_{g}=\right.$ $70{ }^{\circ} \mathrm{C}$ ), chain segments are highly mobile and likely to disentangle as soon as they are not trapped between two crystals. Thus, only disentanglement of dangling chain-ends is possible. When a pull-out event occurs for a $\mathrm{HB}$ in which the dangling-end is anchored, the latter grow. The chain segment previously trapped now has the possibility to disentangle.

This concept is illustrated in Figure 10. As the strain increase, more pull-out events occur, which translates into a progressive decrease of the number of crystallized HB per chain. As a consequence, the length of the dangling-ends increases as follows:

$M_{\text {dang }}=\frac{M-<N_{\text {crys }}>(1-f)\left(<L_{n}>M_{0, P B T}\right)}{1+<N_{\text {crys }}>(1-f)}$

Where $f$ is an independent parameter representing the fraction of HBs pulled out from the crystal domains during deformation with $f$ given by the number of HB pulled out divided by the number of $\mathrm{HB}$ per chain initially crystallized $\left\langle N_{\text {crys }}\right\rangle$. The weight fraction of a 
chain being part of a dangling-end $(2 x$ $M_{\text {dang }} / M$ ) contributes less to the stress response because it is able to disentangle. For a given $f$, this is higher for the low-Mw samples (Figure 11(a)). As the $\mathrm{Mw}$ increases, the number of dangling ends decreases as $\propto 1 / M$, while their length increases as $\sim M /(1+M)$.
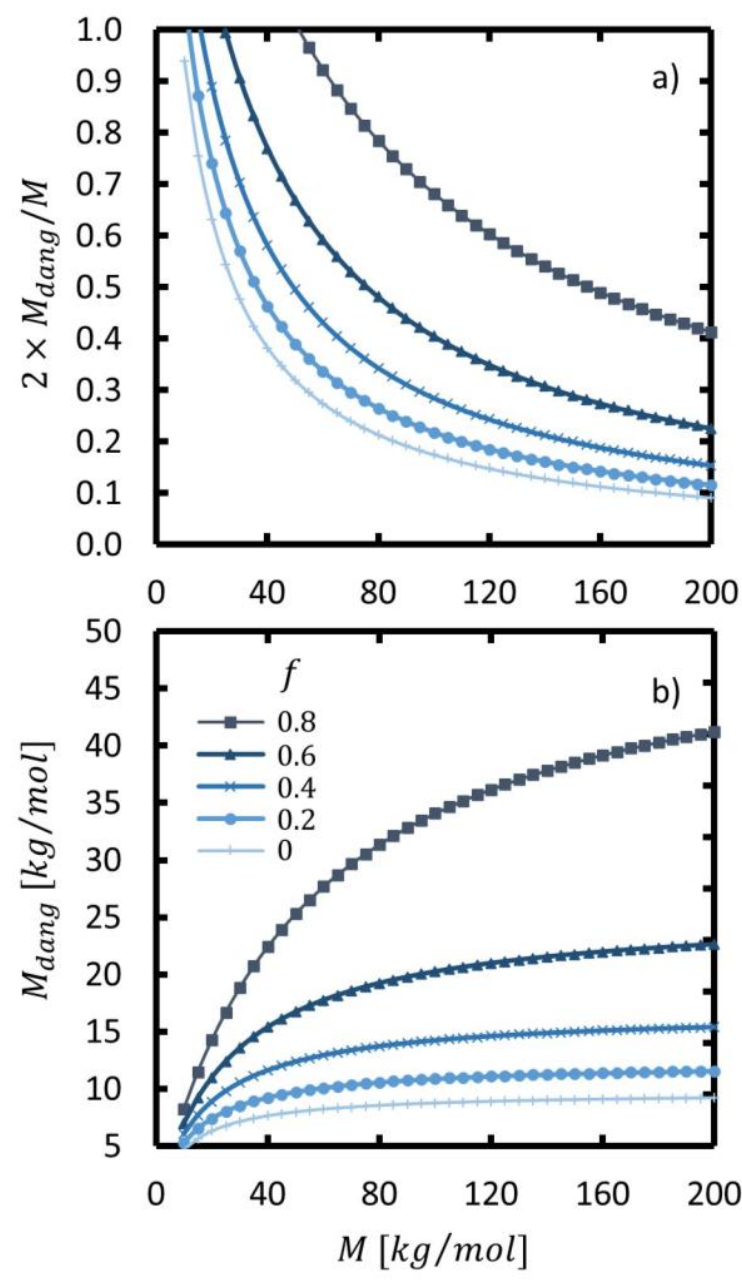

FIGURE 11 a) Weight fraction of dangling-end in a chain, i.e. not trapped between two crystalline HBs, and b) Dangling-ends length as a function of the total chain mass, $M$. Both the plots show how these vary with increasing the fraction of HBs pulled-out during deformation (f).
Thus, as shown in Figure 11(b) the dangling end length initially increases with total $\mathrm{Mw}$ before plateauing. Also when no pull-out has occurred $(f=0)$, the dangling-end mass does not strongly depend on Mw but the dependency grows with increasing $f$, i.e. as the pull-out events occur during deformation.

The dangling-end length becomes crucial when the dynamics of disentanglements are important for the determination of the stress response in the system. Indeed, these chain segments cannot simply disentangle by reptation due to the constrains imposed by the PBT crystals. By modelling a dangling-end anchored to a PBT crystal as a branch of a star polymer, the longest time for the branch to relax from its original constraints (retraction time) grows exponentially with its mass. ${ }^{49}$ It is calculated by

$\tau_{\text {retraction }}=\tau_{e}(\mathrm{~T}) \exp \left(\frac{15}{8} \frac{M_{\text {dang }}}{M_{e}}\right)$

$\tau_{e}(\mathrm{~T})$ is calculated from $\tau_{e}\left(240^{\circ} \mathrm{C}\right)=2.4 \times 10^{-8} \mathrm{~s}$ obtained from a theoretical fit to experimental data from melt rheology ${ }^{50}$ Arrhenius-scaled with an activation energy of $\mathrm{Ea}=5.24 \times 10^{4}$ $\mathrm{J} / \mathrm{mol}$. The estimated retraction times at the testing temperatures are of the same order of the time scales of our experiments, $1 / \dot{\varepsilon}$ (Figure 12(a)). Note also that as the $\mathrm{Mw}$ increases the relative increase in retraction time decreases, e.g. the increase in retraction time from 25 to $50 \mathrm{~kg} / \mathrm{mol}$ is much larger than between 100 and $150 \mathrm{~kg} / \mathrm{mol}$ at a given $f$.

To calculate the number of SBU, we exclude the entanglements present in the dangling chainsegments with a retraction time shorter than $1 / \dot{\varepsilon}$ since they are able to disentangle and relax during deformation. Consequently, they do not 
contribute to the stress response. The SBUs molar density is then calculated by

$$
\begin{aligned}
& \operatorname{SBU}(f)=\left[1-f_{\text {dang }}(f)\right]\left(\frac{\rho}{M_{e}}\right) \\
& +(1-f)<N_{\text {crys }}>\left(\frac{\rho}{M}\right)
\end{aligned}
$$

a)

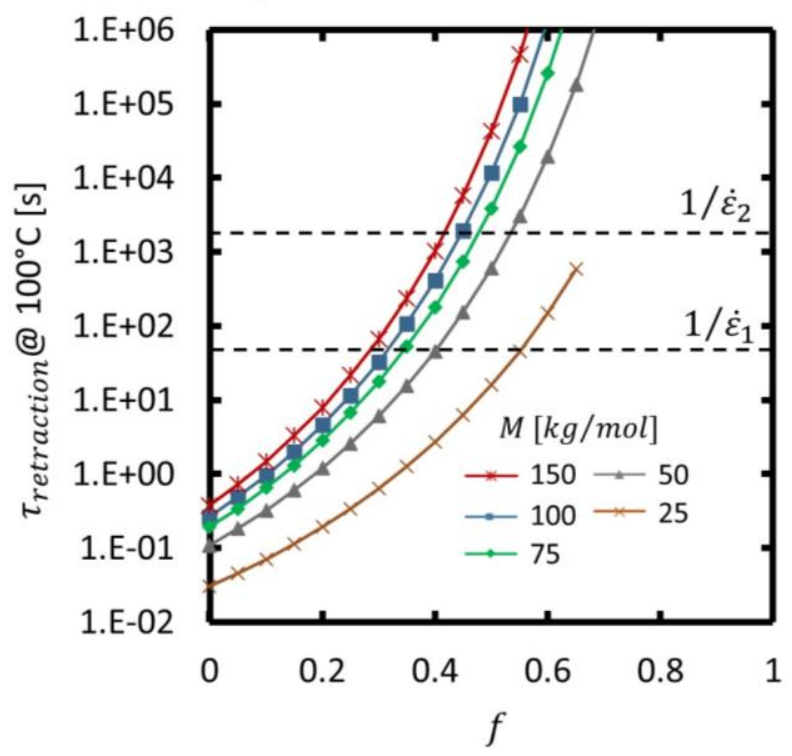

where $f_{\text {dang }}(f)=2 \times\left(M_{\text {dang }}(f)\right) / M$ is the weight fraction of dangling-ends in a chain and polymer density, $\rho=1.1 \mathrm{~g} / \mathrm{cm}^{3}$. b)

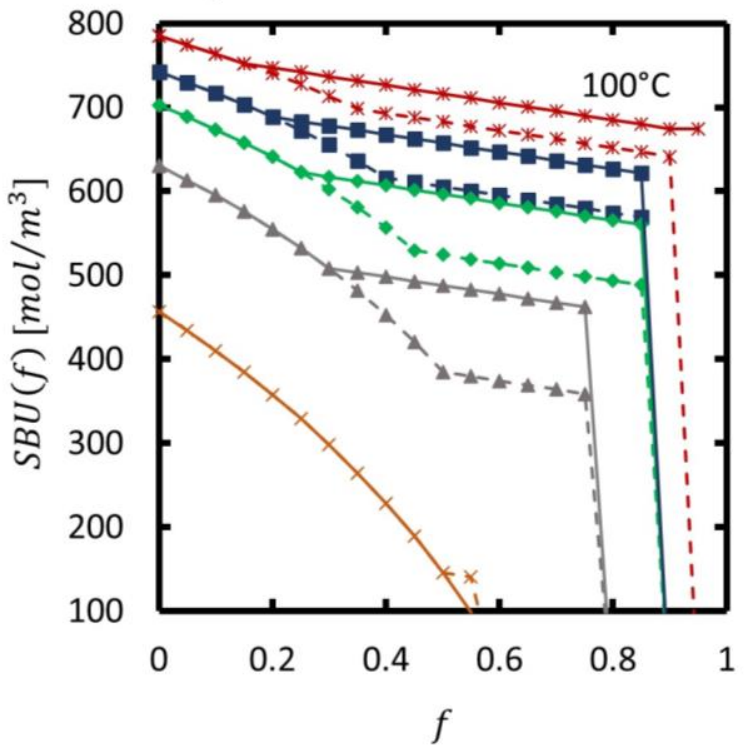

GURE 12 a) Retraction time at $100{ }^{\circ} \mathrm{C}$ of a dangling-end modelled as a branch of a star polymer versus the fraction of pulled-out HBs $(f)$. The curves end when $<$ Ncrys $>(1-f)<1$ and the retraction time cannot be defined, i.e. when, on average, the chains no longer have at least one HB in a crystal and are thus free to relax via reptation. b) Molar density of the SBUs in the system as a function of $f$. Solid and dashed lines correspond to the experimental time scales for the low and high strain-rates used, $\dot{\varepsilon}_{1}=$ $0.17 \mathrm{~s}^{-1}$ and $\dot{\varepsilon}_{2}=0.0017 \mathrm{~s}^{-1}$. Different colors and symbols correspond to the different chain lengths, $M$, given in the legend.

If $f_{0}$ is the fraction of pulled out HBs at which the dangling-ends are not able to relax anymore, i.e. $\tau_{\text {retraction }}>1 / \dot{\varepsilon}$, at $f \geq f_{0}$ the first term of Equation 8 becomes [ $\left.1-f_{\text {dang }}\left(f_{0}\right)\right]\left(\rho / M_{e}\right.$ ). This is equivalent to saying that the fraction of dangling-end that at $f<f_{0}$ was able to relax is still able to do so.

Figure 12(b) shows the molar density of SBUs for different $M$, plotted against the fraction of pulled out HBs during deformation. In line with our experimental results (Figure 9), this model predicts that for low-Mw chains, lowering the strain-rates leads to fewer SBUs compared to high strain-rates and the effect of rate is less as $M$ increases. Note that for short chains, almost no strain-rate effect is predicted in this strainrate range as dangling-ends are always short enough to disentangle. For intermediate length, we observe the largest influence of strain-rate. At high $\mathrm{Mw}$, the rate effect is decreased. This is because high-Mw samples show more SBUs at 
each stage of deformation which results in a higher stress response (strain-hardening), network elasticity and connectivity in agreement with the experimental observations from the tensile and cyclic tests. In summary, increasing $\mathrm{Mw}$ decreases the number of dangling-ends and chain weight fraction belonging to a dangling-end. However, the absolute length of the dangling-ends increases slowing down the disentanglement kinetics.

Consequently, at each stage of deformation high-Mw samples have more SBUs and a less time-dependent stress response than low-Mw samples. This approach is a simplification that does not take into account the poly-dispersity of the chain-length or single blocks. The model treats $f$ as an independent parameter while in reality it should depend on the applied strain. However, it is reasonable to believe that the strain dependency of $f$ should not be $\mathrm{Mw}$ independent as Mw should influence the pullout rate of the HBs. It is not trivial to assess whether the increase in $\mathrm{Mw}$ leads to a faster or slower pull-out rate. Longer chains are better connected with the network, which also means that they have more sites susceptible to the pull-out mechanism and which are under stress for longer time during deformation. This would lead to the pull-out rate increasing with $\mathrm{Mw}$. On the other hand, the pull-out event has a higher chance to cause a low-Mw chain to become disconnected from the network. ${ }^{27}$ This would generates higher stresses locally facilitating the pull-out of the neighboring HBs and generating a knock-on effect that would accelerate the pull-out rate of the HB for low-Mw systems. Despite the simplifying assumptions and unanswered questions about the link between strain, $f$, and $\mathrm{Mw}$, this model qualitatively identifies a plausible physical mechanism able to explain the observed effects of $\mathrm{Mw}$ and strain-rate.

\section{CONCLUSIONS}

The presented series of systematic experimental observations combined with a simple mechanistic model bring new insights furthering the understanding of the effect of temperature, $\mathrm{Mw}$ and composition on the mechanical properties of soft-TPEs. The simple model provides a plausible mechanism driving the $\mathrm{Mw}$ and strain-rate effects on the mechanical response that is consistent with, and builds upon, previous studies..$^{8,19,25,27}$ Key insights are summarized below:

The initial modulus is determined by the ribbon-like PBT crystal structure and volume fraction and increases with the wt\% of PBT as a result of increased PBT crystallinity. Upon reloading after moderate deformation ( $<200 \%$ strain), the modulus and hysteresis decrease while the tensile set remains relatively constant. This suggests that the initial loading response is dominated by a rigid, loading bearing crystal structure that breaks up with deformation and whose unloading behavior is dominated by the amorphous regions.

Whether or not PTMO SIC occurs has a large influence on the high strain mechanical response and depends on the testing temperature. Failure strain and stress do not necessarily scale with the wt\% of PBT if PTMO SIC occurs. SIC strongly increases the strain hardening response and decreases the elastic recovery at high strains. The amount of SIC increases with increasing amount and length of PTMO soft block while increasing Mw decreases the onset strain for SIC. 
Temperature resistance is not governed by the distance of the testing temperature from the peak melting temperature. Network connectivity contributes strongly, and temperature resistance increases with increasing $\mathrm{Mw}$.

- While Mw has little influence during continuous loading up to $\sim 300 \%$ strain, increasing $\mathrm{Mw}$ increases the strain-hardening response at higher strains as well as the elastic recovery at low and high strains. At higher temperatures, where no SIC occurs, increasing the strain rate increases the strain hardening of low-Mw more than high-Mw samples, while increasing the failure strain of both.

- A model based on the network connectivity as generated by both the crystalline PBT segments and the entangled chains is proposed to explain the observed effects of $\mathrm{Mw}$ on the rate and temperature dependent characteristic of the strainhardening response. This model shows how the weight fraction and length of the dangling-ends vary as a function of chain length and number of HB pull-out events. The number of SBUs evolves with pull-out events as well as with the time-scale of dangling-end relaxation. For long dangling-ends, disentanglement time is longer than the experimental time scale and entanglements in the dangling-ends contribute to the stress response.

\section{ACKNOWLEDGEMENTS}

This work was financially supported by funding from the H2020 Programme (MARIE SKŁODOWSKA-CURIE ACTIONS) of the European Commission's Innovative Training Networks (H2020-MSCA-ITN-2017) under DoDyNet REA Grant Agreement $\mathrm{N}^{\circ} .765811$. The authors thankfully acknowledge Louis Pitet and the polyester group in DSM for the synthesis of the model systems, Mingwen Tian and Junyu $\mathrm{Li}$ respectively for the support with AFM and X-ray images acquisition and Patrick van Soelen and Marcel Teeuwen for the help with the mechanical instrumentation. Finally, the authors also acknowledge EVR is Research Associate of the FNRS.

\section{REFERENCES AND NOTES}

1. Fakirov, S. in Handbook of Condensation Thermoplastic Elastomer; Wiley-VCH Verlag $\mathrm{GmbH}$ and Co, Weinheim; 2005.

2. Stevenson, J. C., Cooper, S. L. Macromolecules 1988, 21, 1309-1316.

3. Nébouy, M.; De Almeida, A.; Brottet, S.; Baeza, G. P. Macromolecules 2018, 51 (16), 6291-6302.

4. Gabriëlse, W.; Soliman, M.; Dijkstra, K. Macromolecules 2001, 34 (6), 16851693.

5. Litvinov, V. M.; Bertmer, M.; Gasper, L.; Demco, D. E.; Blümich, B. Macromolecules 2003, 36 (20), 75987606.

6. De Almeida, A.; Nébouy, M.; Baeza, G. P. Macromolecules 2019, 52 (3), 12271240.

7. Sijbrandi, N. J. Kimenai, A. J.; Mes, E. P. C.; Broos, R.; Bar, G.; Rosenthal, M.; Odarchenko, Y.; Ivanov, D. A.; Dijkstra, P. J.; Feijen, J. Macromolecules 2012, 45 (9), 3948-3961.

8. Zhu, P.; Dong, X.; Wang, D. Macromolecules 2017, 50 (10), 3911-3921.

9. Smith, T. L. J. Polym. Sci. Part B Polym. Phys. 1974, 12 (9), 1825-1848.

10. Gaymans, R. J. Prog. Polym. Sci. 2011, 36 (6), 713-748.

11. Seefried, C. G.; Koleske, J. V.; Critchfield, F. E. J. Appl. Polym. Sci. 1975, 19 (9), 2503-2513.

12. Fakirov, S.; Apostolov, A. A.; Boeseke, P.; Zachmann, H. G. J. Macromol. Sci. Part B. 1990, 29 (4), 379-395.

13. Konyukhova, E. V.; Neverov, V. M.; Godovsky, Y. K.; Chvalun, S. N.; 
Soliman, M.. Macromol. Mater. Eng. 2002, 287 (4), 250-265.

14. Van Bogart, J. W. C.; Lilaonitkul, A.; Lerner, L. E.; Cooper, S. L. J. Macromol. Sci. Part B 1980, 17 (2), 267-301.

15. Deschamps, A. A.; Grijpma, D. W.; Feijen, J. Polymer 2001, 42 (23), 9335-9345.

16. Dahrouch, M.; Schmidt, A.; Leemans, L.; Linssen, H.; Götz, H. Macromol. Symp. 2003, 199, 147-162.

17. Sup Lee, H.; Ra Yoo, S.; Won Seo, S.. J. Polym. Sci. Part B Polym. Phys. 1999, 37 (22), 3233-3245.

18. Biemond, G. J. E.; Feijen, J.; Gaymans, R. J. J. Mater. Sci. 2008, 43 (10), 3689-3696.

19. Nébouy, M.; Louhichi, A.; Baeza, G.,P. J. Polym. Eng. 2019, 40 (9), 715-726.

20. Stribeck, N.; Sapoundjieva, D.; Denchev, Z.; Apostolov, A. A.; Zachmann, H. G.; Stamm, M.; Fakirov, S. Macromolecules 1997, 30 (5), 1329-1339.

21. Stribeck, N.; Fakirov, S.; Apostolov, A. A.; Denchev, Z.; Gehrke, R. Macromol. Chem. Phys. 2003, 204 (7), 1000-1013.

22. Unsal, E.; Yalcin, B.; Yilgor, I.; Yilgor, E.; Cakmak, M. Polymer. 2009, 50 (19), 4644-4655.

23. Nogales, A.; Sics, I.; Ezquerra, T. A.; Denchev, Z.; Balta Calleja, F. J.; Hsiao, B. S. Macromolecules 2003, 36 (13), 4827-4832.

24. Yeh, F.; Hsiao, B. S.; Sauer, B. B.; Michel, S.; Siesler, H. W. Macromolecules 2003, 36 (6), 1940-1954.

25. Niesten, M. C. E. J.; Gaymans, R. J. Polymer. 2001, 42 (14), 6199-6207.

26. Gorce, J. -N; Hellgeth, J. W.; Ward, T. C. Polym. Eng. Sci. 1993, 33 (18), 11701176.

27. Aime, S.; Eisenmenger, N. D.; Engels, T. A. P. J. Rheol. 2017, 61 (6), 1329-1342.

28. Cho H.; Mayer S.; Poselt E.; Susoff M.; in 't Veld P. J.; Rutledge G. C.; Boyce M. C., Polymer 2017, 128, 87-89.

29. Bowman, I.; Brown, D.; Wetton, R. Polymer 1969, 10,715-718.
30. Yokouchi, M.; Sakakibara, Y.; Chatani, Y.; Tadokoro, H.; Tanaka, T.; Yoda, K. Macromolecules 1976, 9, 266-273.

31. Niehaus, D.E.; Jackson, C., Polymer 2000, 41, 259-268.

32. Das, C.; Read, D., J. ; Kelmanson, M., A.; Mcleish, T.C.B. Phys. Rev. E. 2006, 74 (1).

33. Seitz, M. E.; Burghardt, W. R.; Shull, K. R., Macromolecules 2009, 42 (22), 91339140.

34. Deplace, F.; Scholz, A. K.; Fredrickson, G. H.; Kramer, E. J.; Shin, Y. W.; Shimizu, F.; Zuo, F.; Rong, L.; Hsiao, B. S.; Coates, G. W., Macromolecules 2012, 45 (13), 5604-5618.

35. Deplace, F.; Wang, Z.; Lynd, N.; Hotta, A.; Rose, J.; Hustad, P.; Tian, J.; Ohtaki, H.; Coates, G.; Shimizu, F.; Hirokane, K.; Yamada, F.; Shin, Y. W.; Rong, L.; Zhu, J.; Toki, S.; Hsiao, B.; Fredrickson, G.; Kramer, E., J. Polym. Sci. Part B Polym. Phys. 2010, 48 (13), 1428-1437.

36. Baeza, G. P. Macromolecules 2018, 51 (5), 1957-1966.

37. Gibson, L.J. and Ashby, M.F. in Cellular Solids: Structure and Properties, second ed.; Cambridge University Press, Cambridge; 1997.

38. Schmidt, A.; Veeman, W. S.; Litvinov, V. M.; Gabriëlse, W. Macromolecules 1998, 31 (5), 1652-1660.

39. Rottler, J.; Robbins, M. O. Phys. Rev. E, 2003, 68.

40. Rottler, J.; Robbins, M. O. Phys. Rev. Lett. 2005, 95.

41. Eyring, H. J. Chem. Phys. 1936.

42. Nitta, K. H.; Ishiburo, T. J. Polym. Sci. Part B Polym. Phys. 2002, 40 (18), 2018-2026.

43. Termonia, Y.; Allen, S. R.; Smith, P. Macromolecules 1988, 21 (12), 34853489.

44. Kennedy, M. A.; Peacock, A. J.; Failla, M. D.; Lucas, J. C.; Mandelkern, L, Macromolecules 1995, 28 (5), 14071421.

45. Seguela, R.; Rietsch, F. Polymer. 1986, 27 (5), 703-708. 
46. Kennedy, M. A.; Peacock, A. J.; Mandelkern, L. Macromolecules 1994, 27 (19), 52975310.

47. Men, Y.; Rieger, J.; Strobl, G. Phys. Rev. Lett. 2003, 91 (9).

48. Bartczak, Z.; Galeski, A. Macromol. Symp. 2010, 294 (1), 67-90.
49. Watanabe, H. Prog. Polym. Sci. 1999, 24 (9), 1253-1403.

50. Likhtman, A. E.; McLeish, T.C.B. Macromolecules 2002, 35, 6332-6343.

\title{
Supplemental Information
}

\section{Morphological origins of temperature and rate dependent mechanical properties of model soft thermoplastic elastomers}

\author{
Simone Sbrescia ${ }^{1}$, Jianzhu Ju${ }^{2}$, Tom Engels ${ }^{1}$, Evelyne Van Ruymbeke ${ }^{3}$, Michelle Seitz ${ }^{1}$ \\ ${ }^{1}$ DSM Materials Science Center, Urmonderbaan, 6167 RD Geleen The Netherlands \\ ${ }^{2}$ Laboratoire Sciences et Ingénierie de la Matière Molle, ESPCI Paris, PSL University, CNRS, Sorbonne \\ Université, 75005 Paris, France \\ ${ }^{3}$ Bio and Soft Matter Division (BSMA), Institute of Condensed Matter and Nanosciences (IMCN), \\ Université Catholique de Louvain, B-1348 Louvain-la-Neuve, Belgium;
}

Correspondence to: Michelle Seitz (E-mail: michelle.seitz@dsm.com)

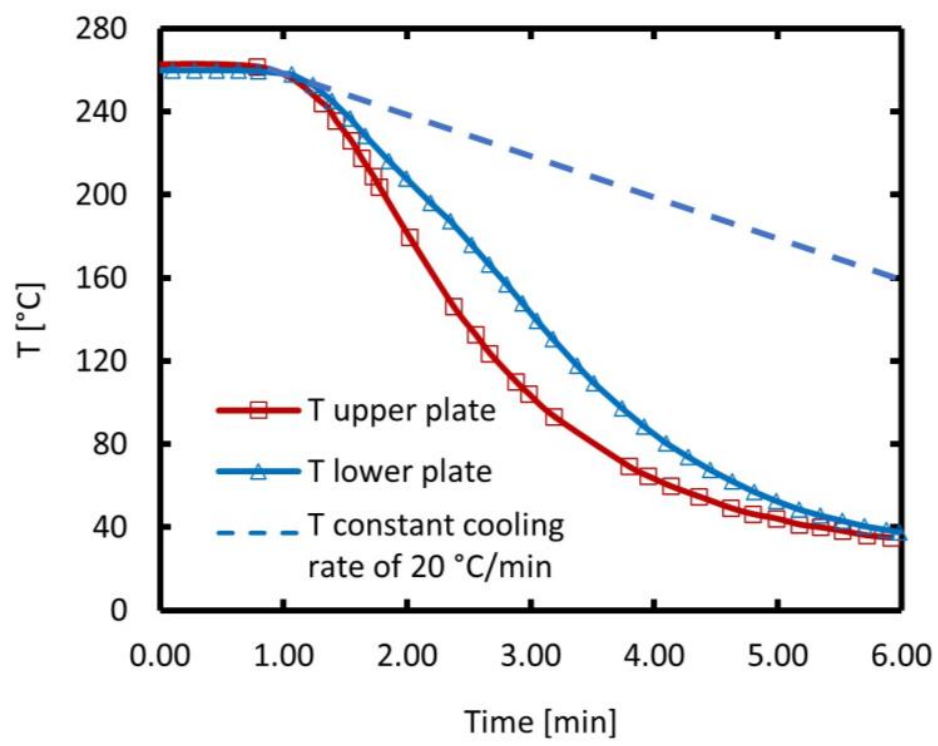

Figure S1 Examples of cooling curves relative to the upper and lower metal plates of the hot-press.

Continuous lines are exported from the hot-press used for the majority of the samples $\left(\sim 40{ }^{\circ} \mathrm{C} / \mathrm{min}\right)$ and 
are relative to an analogous sample preparation at slightly higher temperatures. The dashed curve corresponds to a constant cooling rate of $20^{\circ} \mathrm{C} / \mathrm{min}$, used for some of the samples in this study and are denoted by “*”.

a)

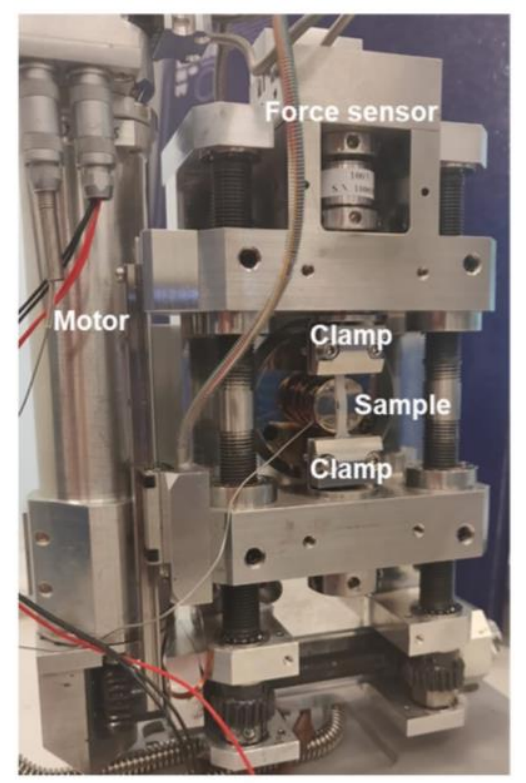

b)

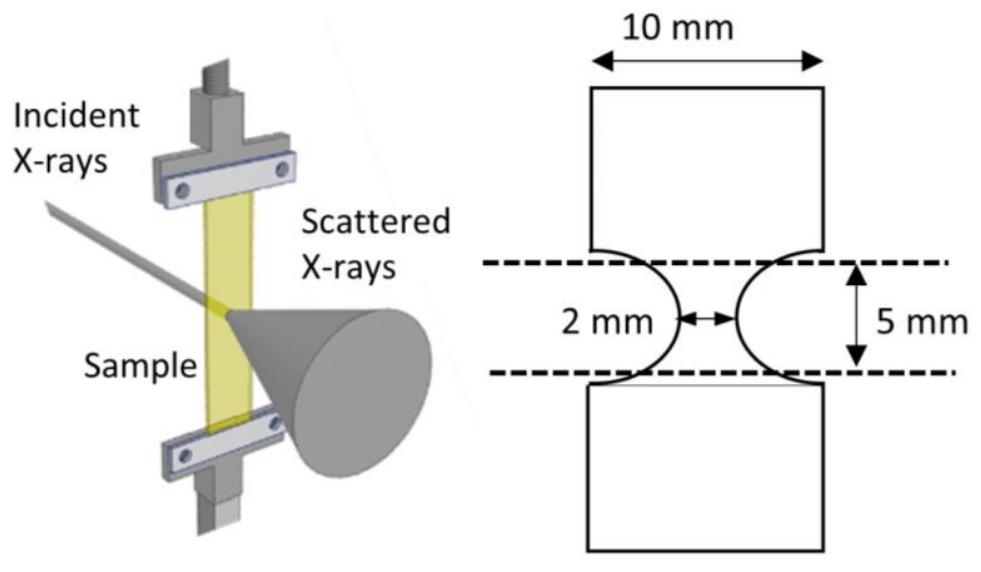

URE S2 a) The tensile stage for X-ray scattering testing and the testing geometry. Extension is in vertical direction. b) Sample's geometry. The sample is obtained by cutting a stripe out of a hot-pressed $200 \mu \mathrm{m}$ film and by reducing the central width with a punch to obtain the geometry shown. This was done to create a dog-bone like geometry to avoid the slippage from the grips during the test.

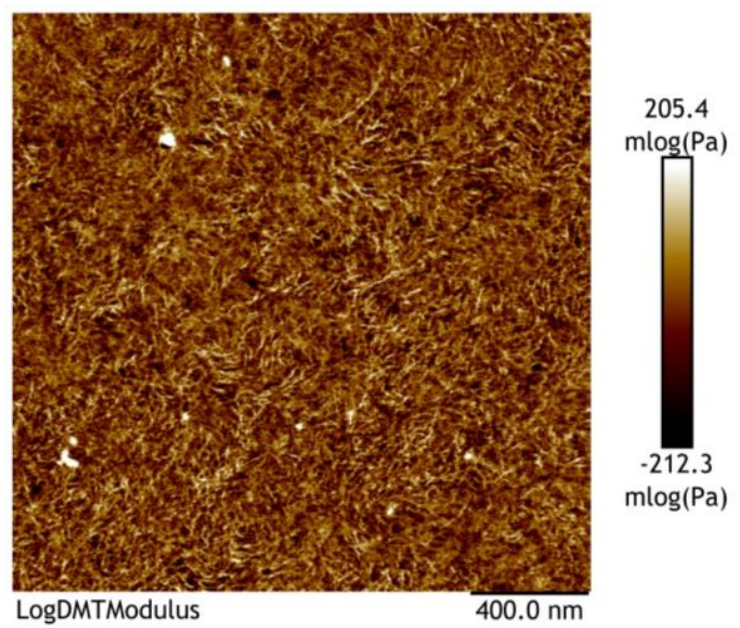

Figure S3 AFM micrograph (QNM) showing the cross-section of a hot-pressed 70_PTMO2k_33 (1 mm thick plate). Brighter regions correspond to a higher elastic modulus (PBT crystals). The differences in structures with figure 1 come from the fact that in cross-section case the sample is isotropic and the 
crystalline ribbons can go across the plane observed with AFM. Whereas the top surface image shows an orthotropic structure where the crystals lay on a single plane, as forced to do due to the hot-press procedure and as address in a previous work. ${ }^{3}$
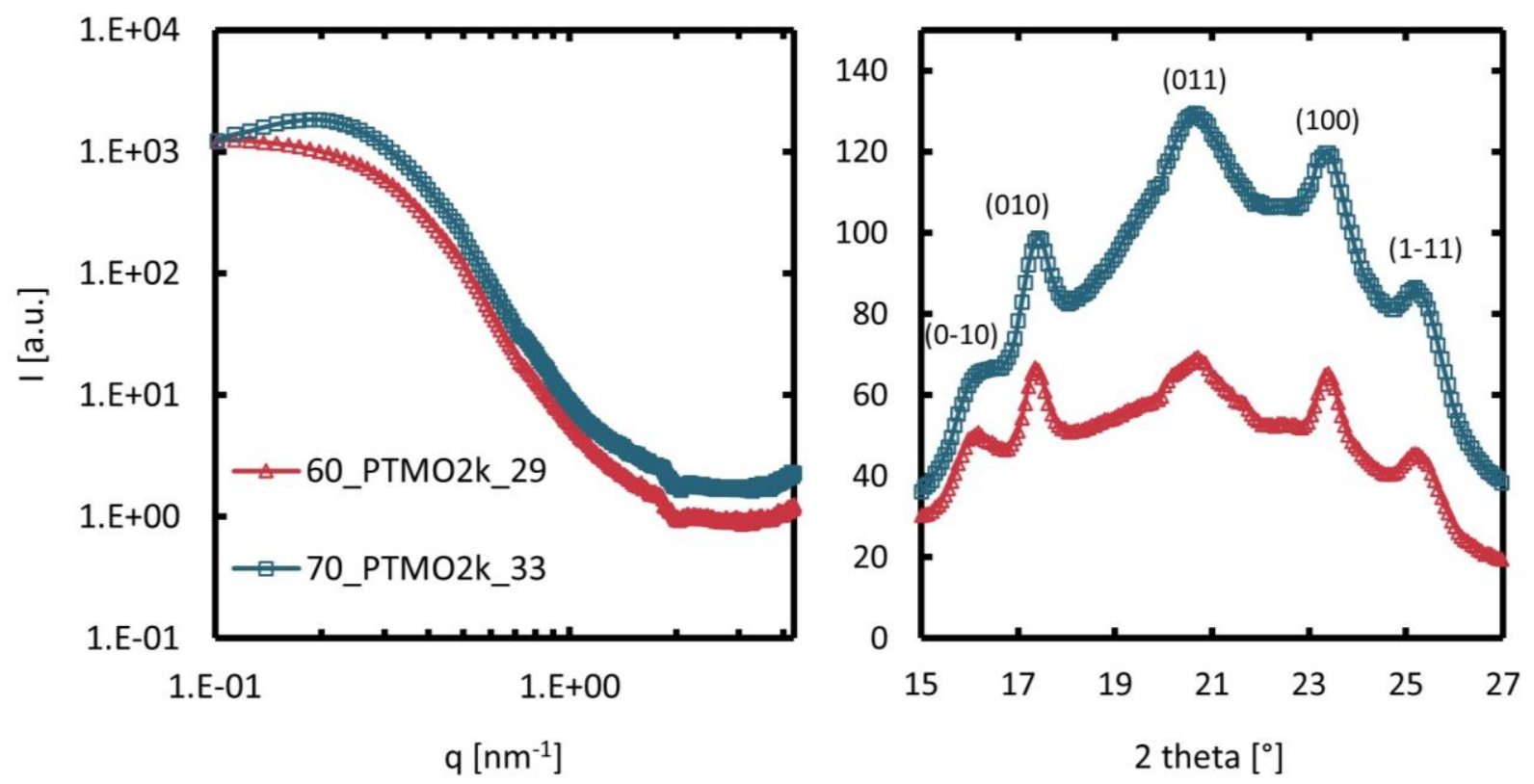

IGURE S4 Average intensities for the samples 60_PTMO_29 and 70_PTMO_33 in the undeformed state.
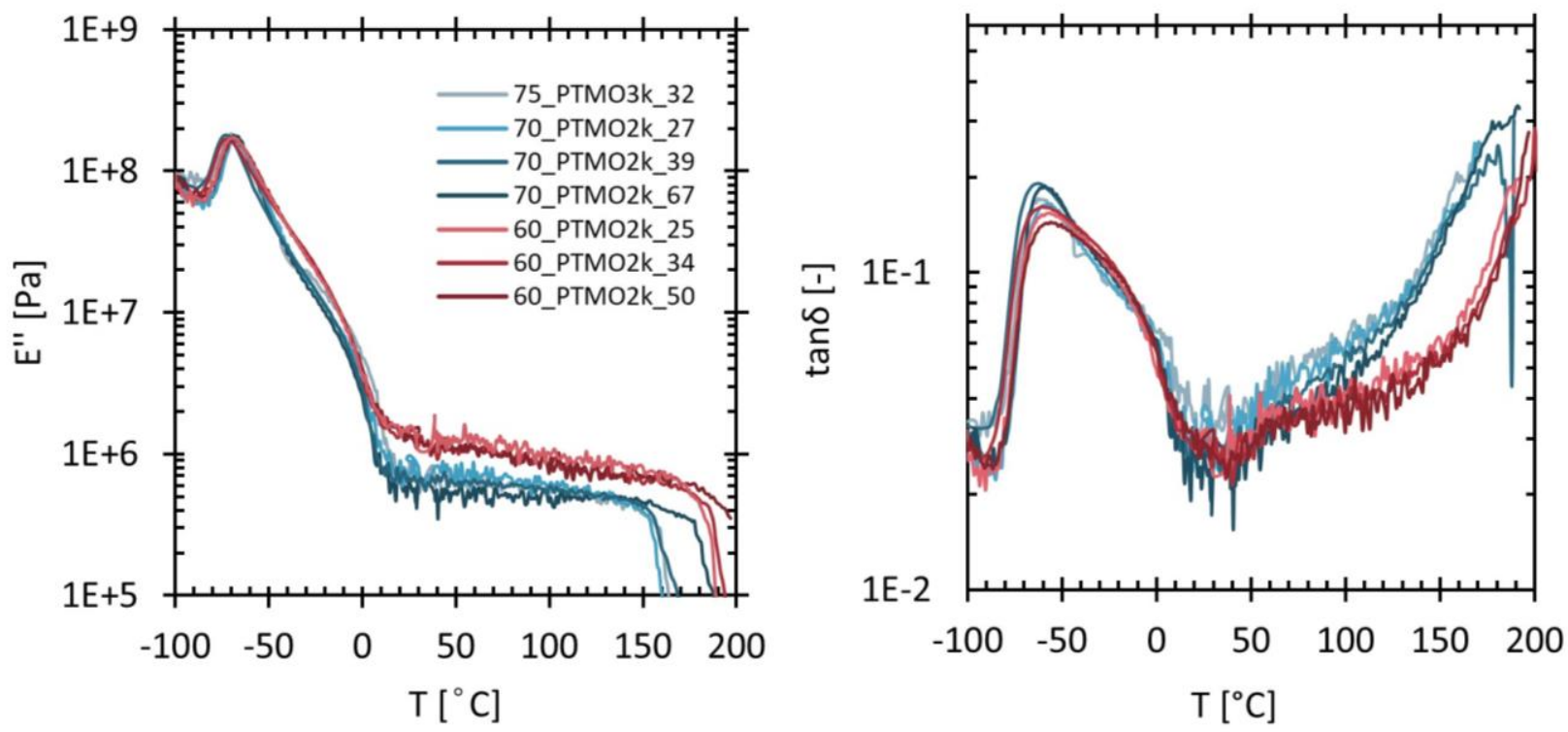

Figure S5 Loss modulus (left) and tand (right) for the series of different $M_{n}$ of samples 60_PTMO2k, 
70_PTMO2k and 75_PTMO3k. $M_{n}$ increases from light to dark. Storage modulus for these samples are shown in main text Figure 2.
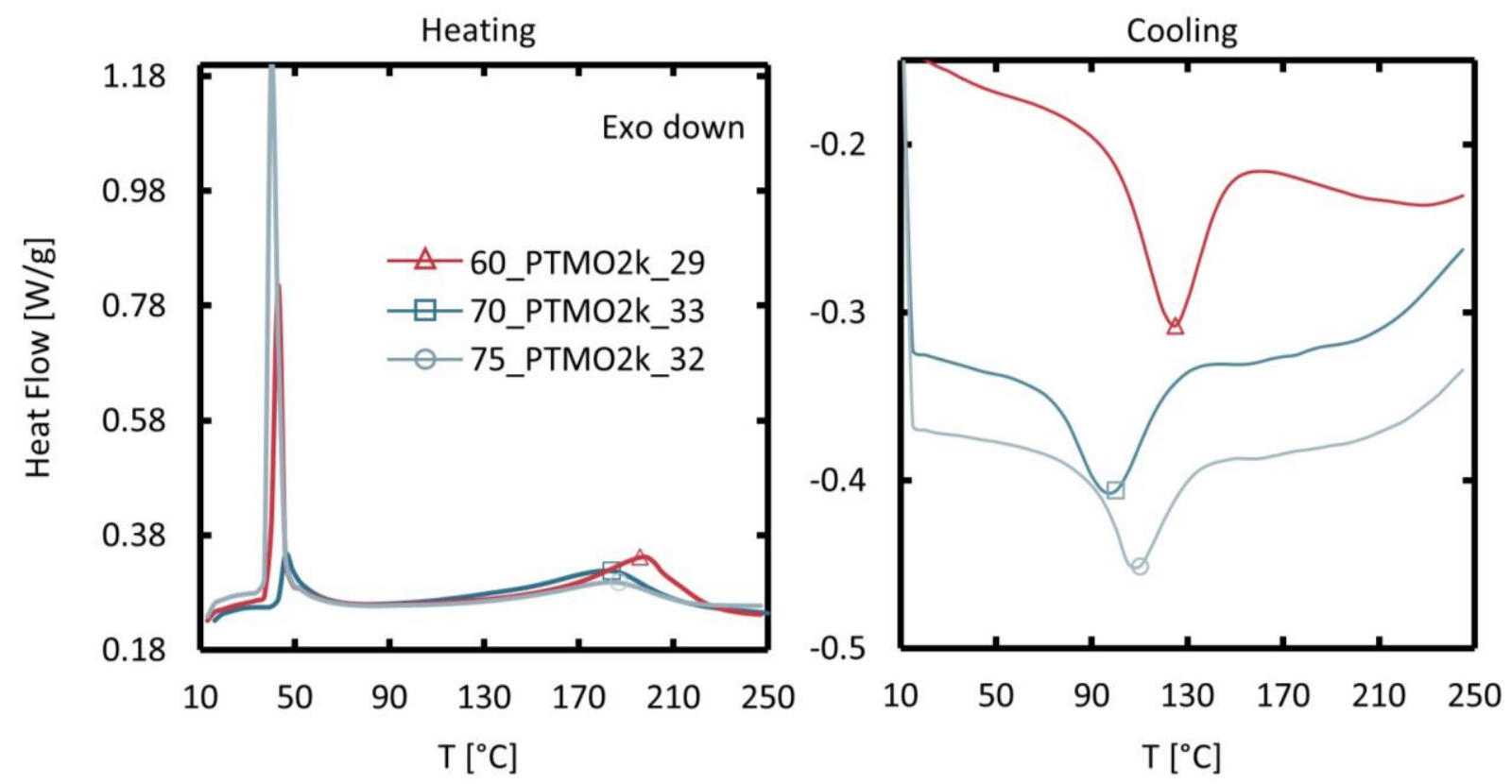

$\mathrm{F}$

igure S6 Left) DSC traces performed near the failure location from samples tested to failure in tension at RT. The peaks between $40^{\circ} \mathrm{C}$ and $50^{\circ} \mathrm{C}$ are attributed to melting of the PTMO SIC, as also detected by Zhu et al. ${ }^{8}$ for polyetherimide. The shape of the peak is probably influenced by the exothermic relaxation of the SB when the PTMO SIC melts. Right) Cooling curves of as-pressed, samples. Symbols indicate the respective PBT melting and crystallization peak maxima. Corresponding first and second heating curves for the un-stretched samples are shown in the main text Figure 3. 

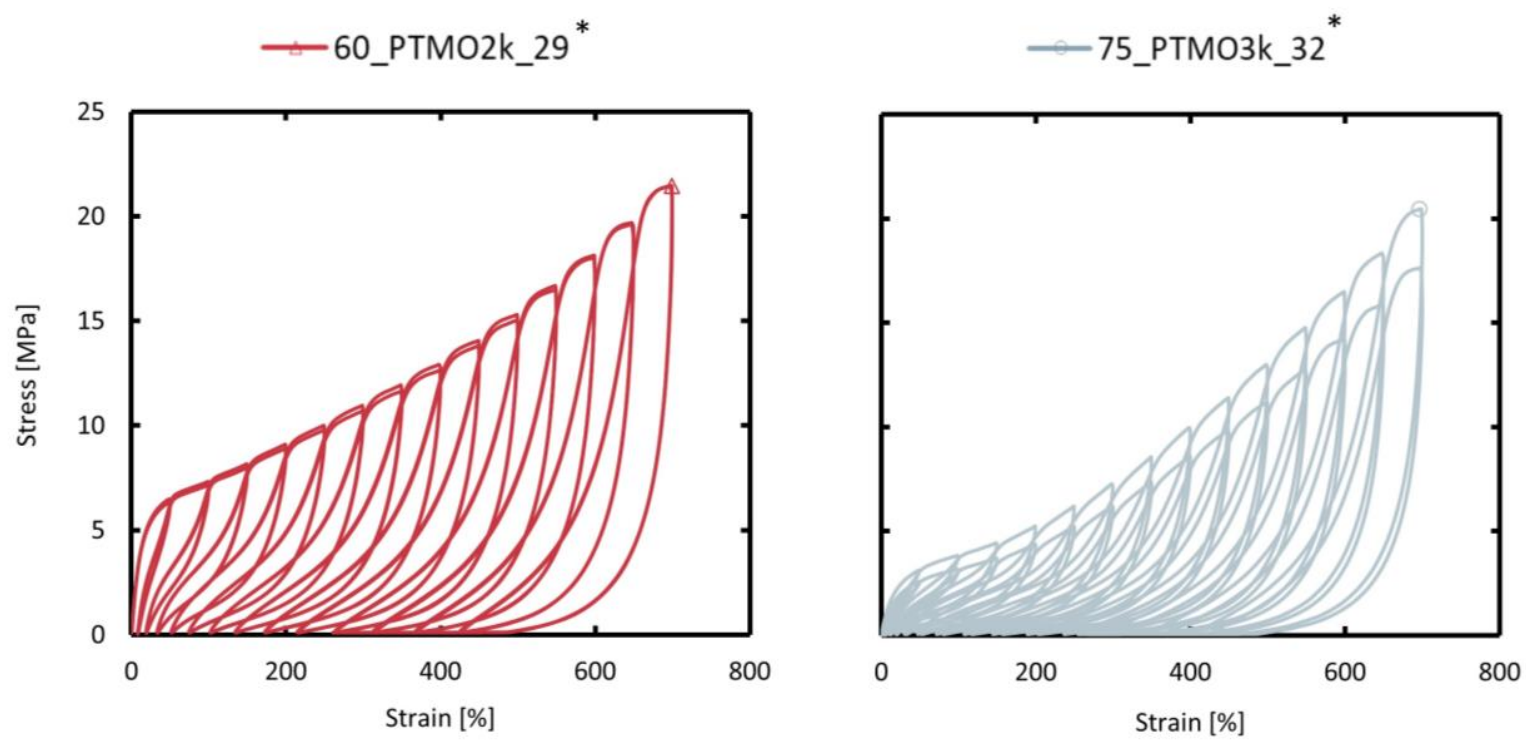

Figur

e S7 Engineering stress-strain cyclic curves, performed at RT and $0.017 \mathrm{~s}^{-1}$ of strain-rate and as described in the Material and Methods section, from which the elastic moduli are calculated at different cycles (Figure 5). Duplicates are shown.
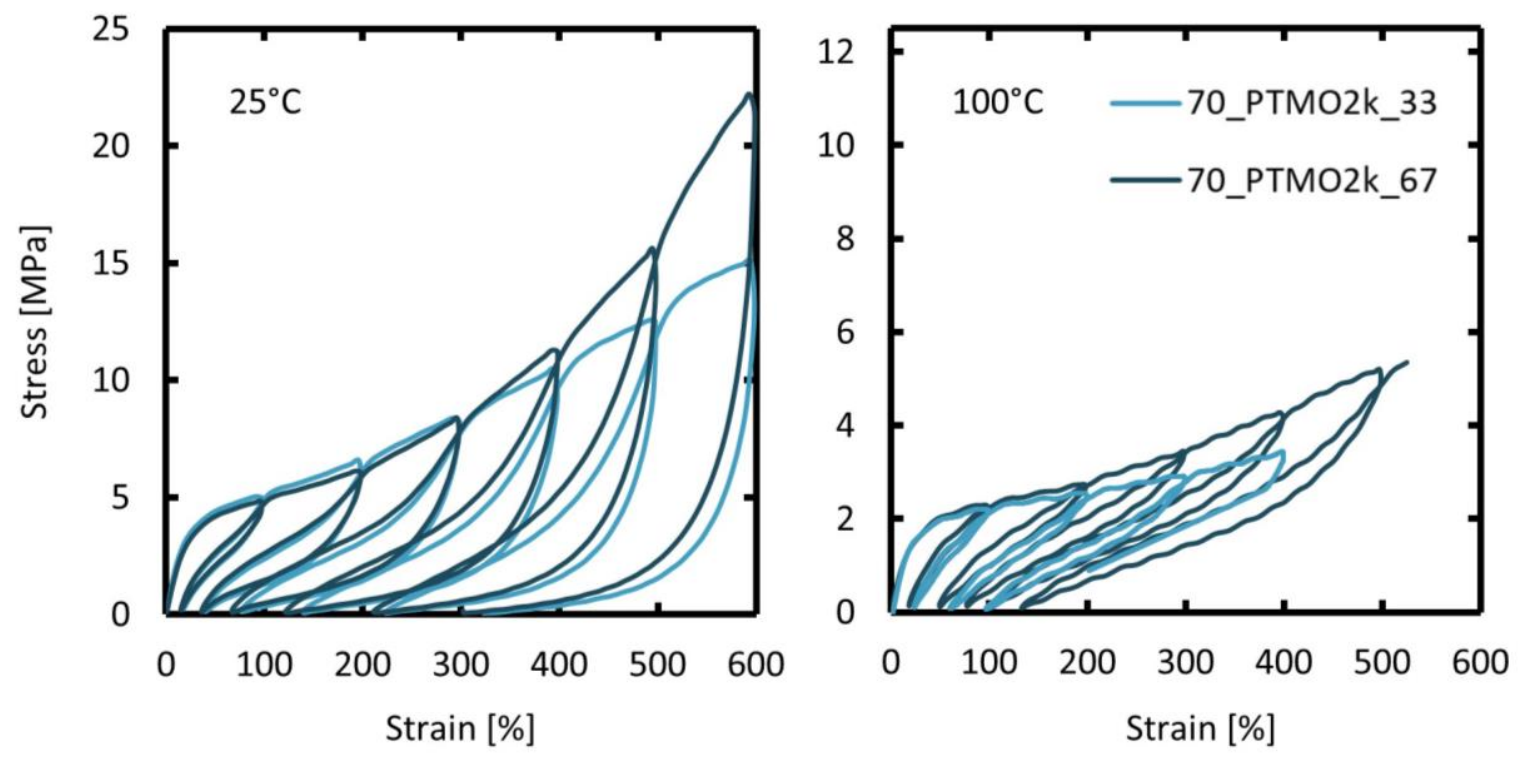

Figur

e S8 Engineering stress-strain cyclic curves, performed at RT and $100{ }^{\circ} \mathrm{C}$ at the strain-rate of $0.017 \mathrm{~s}^{-1}$, as described in the Material and Methods section, from which the recovered strain and the elastic moduli (at RT) at each cycle are collected (Figure 5 and 8). 


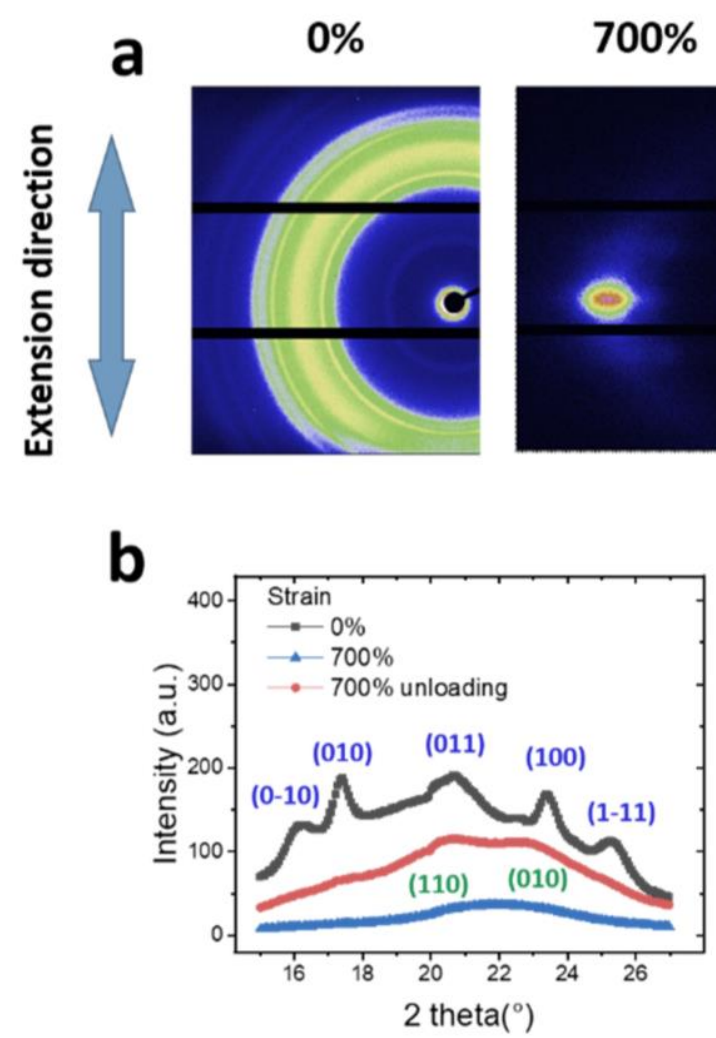

FIGURE S9 (a) WAXS patterns of 60_PTMO2k_29 at strain of $0 \%, 700 \%$ and after unloading from $700 \%$. (b) 1D intensity results of the patterns. PBT crystalline reflections are indicated in blue and PTMO ones are indicated in green.

The WAXS for 60_PTMO2k_29 at strain of 0\%, 700\% and unloaded one from 700\% are shown in Figure S9(a). Patterns intensity are shown in log scale to balance the influence of thickness change with strain. Oriented structure can be observed for $700 \%$ and after unloading the orientation decreases. In the 1D intensity curve for the undeformed sample in Figure S9(b), five peaks can be identified corresponding to PBT crystal reflections. At high strain of $700 \%$, a broad peak can be observed around $2 \theta$ of $22^{\circ}$ and the PBT peaks are too weak to be detected. The crystalline peak of PTMO (110) and (010) are around $21^{\circ}$ and $23^{\circ}$, respectively ${ }^{13,22}$ which is very close to the positions of PBT (011) and (110) peaks. From the decreasing PBT crystalline peak area and the strongly increasing intensity of the broad peak around $22^{\circ}$, we infer small PTMO crystals are formed during stretching while PBT crystal size is decreasing. After unloading from 700\%, PTMO crystalline peaks can still be observed and the two peaks are even clearer. Even though the PTMO crystal is induced by strain, the structure remains stable after fully unloading. 

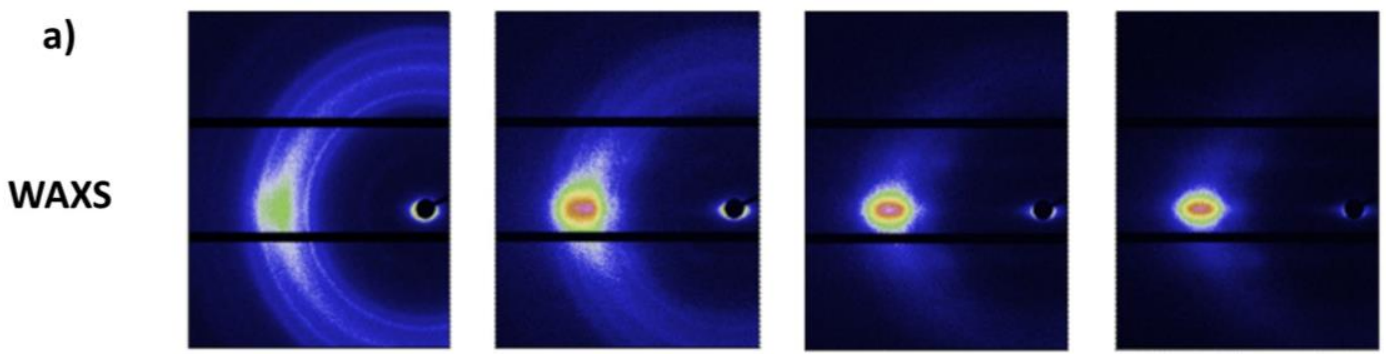

SAXS
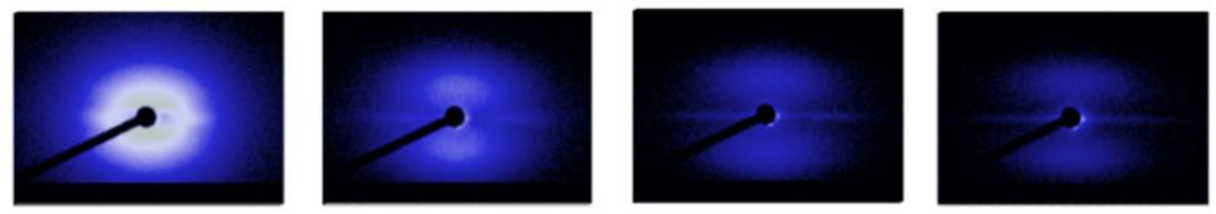

b)
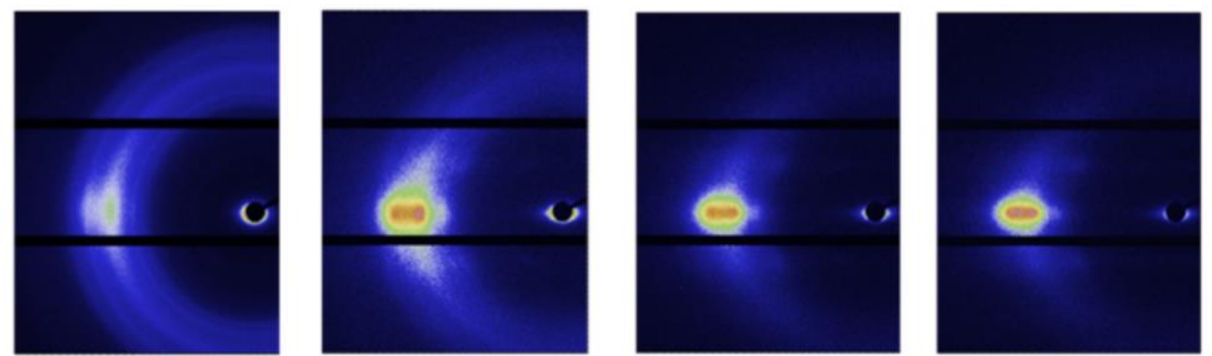

WAXS

SAXS

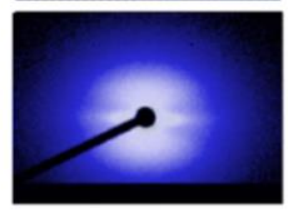

$100 \%$

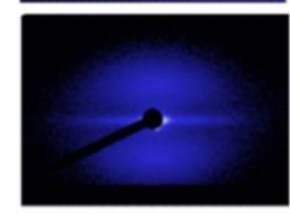

$300 \%$

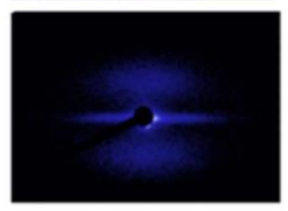

$500 \%$

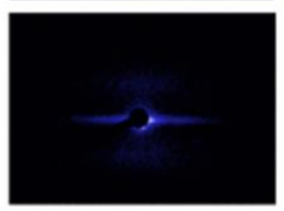

$700 \%$

FIGURE S10 WAXS and SAXS patterns at different applied strains for (a)60_PTMO2k_29 and (b)60_PTMO2k_50. 
a

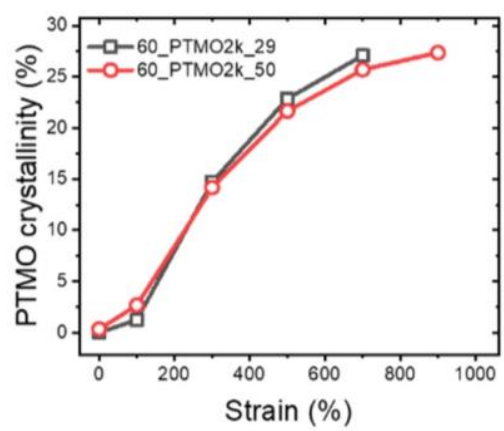

b

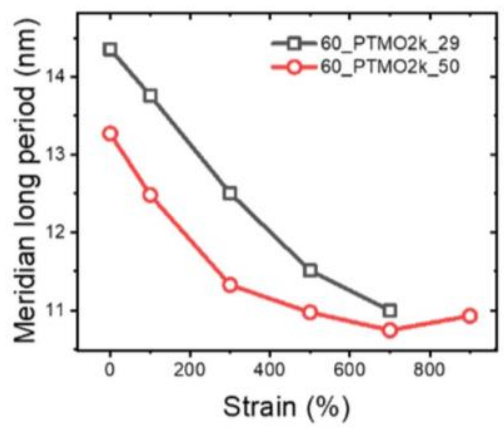

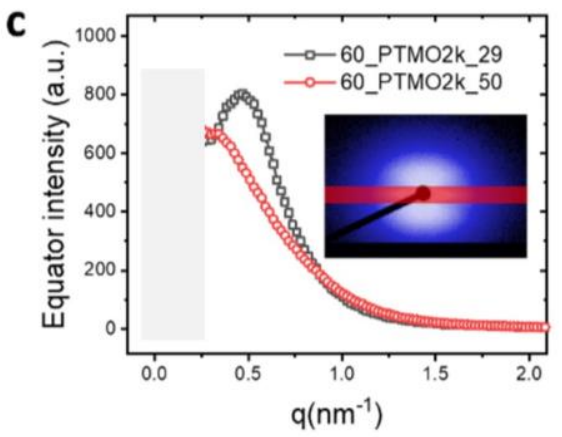

FIGURE S11 (a) PTMO crystallinity and (b) Long periods results at different strain for both samples. (c) 1D intensity in equator direction at strain of $100 \%$. Integration region is indicated in red rectangle. The grey box covers the region of the beam stop.

Figure S10 shows the WAXS and SAXS patterns at different strain for 60_PTMO2k_29 (Figure S10(a)) and 60_PTMO2k_50 (Figure S8(b)). WAXS patterns show a more pronounced orientation for the high-Mw sample. The crystallinity is calculated from the peak areas from 1D WAXS intensity profiles. Results from the integration are shown in Figure S11(a), showing the increase in PTMO crystallinity with increasing strain.

In SAXS, a blob pattern can be detected in the meridian direction, corresponding to a distribution of periodic lamellar structures. The averaged distance between the lamellar (long period) can be calculated from the q value of the 1D equator intensity peak in SAXS (Figure S11(b)), revealing a decrease in long period with increasing strain. The relative intensity of the blob structure dramatically decreases in both materials with increasing strain, a feature recorded also for similar materials. ${ }^{22}$ Besides the blobs, a highly oriented structure in equator direction is observed in all SAXS patterns at high strains. The streak pattern perpendicular to the extension direction in SAXS is normally considered to be a fibril structure, ${ }^{22}$ here assigned to the PTMO SIC. Interestingly, the streak pattern can already be observed at $100 \%$ for 60_PTMO2k_50, while the low-Mw sample shows an intensity maximum in the same direction and at the same level of strain. This is better shown by the equator 1D intensity for the two samples in Figure S11(c). The equator long period at low strain may be due to the lateral distribution of the small broken lamellar. Finally, for 60_PTMO2k_29, streak pattern shows up only from $500 \%$ of strain. 

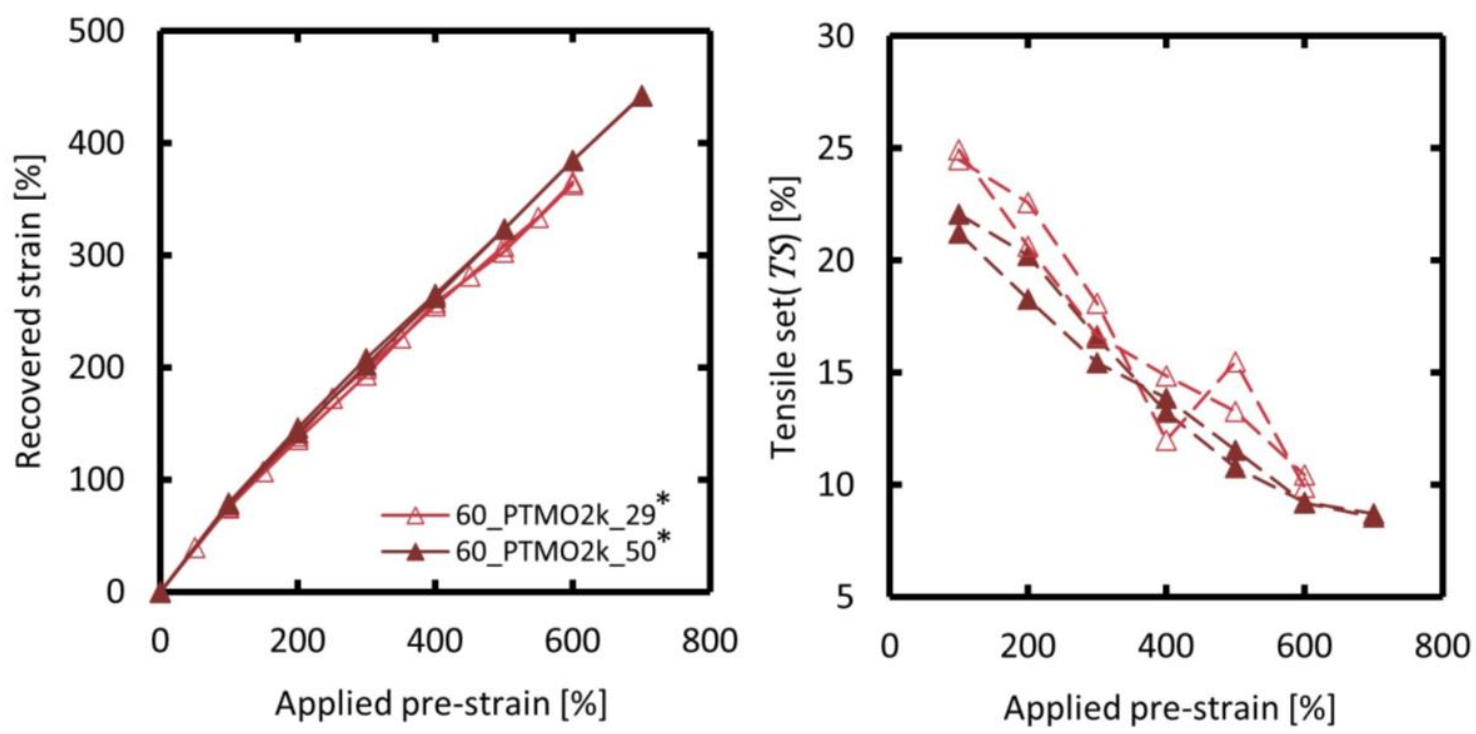

Figure S12 Recovered strain and tensile set relative to the tests performed on 60_PTMO2k_29* and 60_PTMO2k_50* at $100{ }^{\circ} \mathrm{C}$. Analogous conclusions to those in the main text can be made, i.e. higher Mw-samples can recover more strain due to better connectivity. 


\section{GRAPHICAL ABSTRACT}

Simone Sbrescia, Jianzhu Ju, Tom Engels, Evelyne Van Ruymbeke, Michelle Seitz

MORPHOLOGICAL ORIGINS OF TEMPERATURE AND RATE DEPENDENT MECHANICAL PROPERTIES OF MODEL SOFT THERMOPLASTIC ELASTOMERS

Understanding the effects of temperature and time of the mechanical properties of soft thermoplastic elastomers is important to extend their range of applications. Mechanical tests where molecular weight and composition are systematically varied help in understanding what are the different molecular dynamics and key parameters that influence the mechanical behavior. The results are supported by a model that shows the importance of network connectivity in ensuring mechanical temperature resistance.

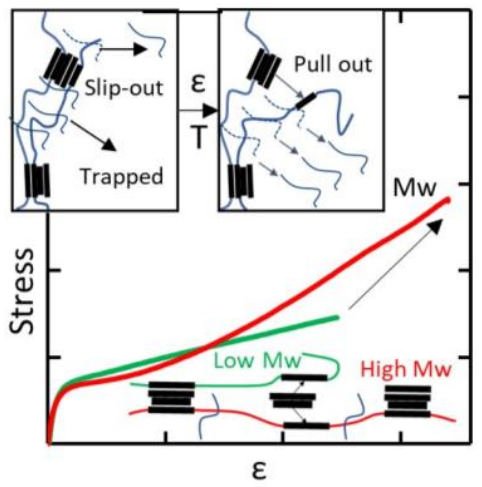

